\title{
How 5000 independent rowers coordinate their strokes in order to row into the sunlight: Phototaxis in the multicellular green alga Volvox
}

\author{
Noriko Ueki ${ }^{1,2}$, Shigeru Matsunaga ${ }^{2,3}$, Isao Inouye ${ }^{2}$, Armin Hallmann ${ }^{1 *}$
}

\begin{abstract}
Background: The evolution of multicellular motile organisms from unicellular ancestors required the utilization of previously evolved tactic behavior in a multicellular context. Volvocine green algae are uniquely suited for studying tactic responses during the transition to multicellularity because they range in complexity from unicellular to multicellular genera. Phototactic responses are essential for these flagellates because they need to orientate themselves to receive sufficient light for photosynthesis, but how does a multicellular organism accomplish phototaxis without any known direct communication among cells? Several aspects of the photoresponse have previously been analyzed in volvocine algae, particularly in the unicellular alga Chlamydomonas.

Results: In this study, the phototactic behavior in the spheroidal, multicellular volvocine green alga Volvox rousseletii (Volvocales, Chlorophyta) was analyzed. In response to light stimuli, not only did the flagella waveform and beat frequency change, but the effective stroke was reversed. Moreover, there was a photoresponse gradient from the anterior to the posterior pole of the spheroid, and only cells of the anterior hemisphere showed an effective response. The latter caused a reverse of the fluid flow that was confined to the anterior hemisphere. The responsiveness to light is consistent with an anterior-to-posterior size gradient of eyespots. At the posterior pole, the eyespots are tiny or absent, making the corresponding cells appear to be blind. Pulsed light stimulation of an immobilized spheroid was used to simulate the light fluctuation experienced by a rotating spheroid during phototaxis. The results demonstrated that in free-swimming spheroids, only those cells of the anterior hemisphere that face toward the light source reverse the beating direction in the presence of illumination; this behavior results in phototactic turning. Moreover, positive phototaxis is facilitated by gravitational forces. Under our conditions, $V$. rousseletii spheroids showed no negative phototaxis.

Conclusions: On the basis of our results, we developed a mechanistic model that predicts the phototactic behavior in $V$. rousseletii. The model involves photoresponses, periodically changing light conditions, morphological polarity, rotation of the spheroid, two modes of flagellar beating, and the impact of gravity. Our results also indicate how recently evolved multicellular organisms adapted the phototactic capabilities of their unicellular ancestors to multicellular life.
\end{abstract}

\section{Background}

The ability of motile organisms to sense changes in external parameters is crucial in finding the best environmental conditions. These tactic responses were already developed in early single-celled life. When multicellular motile organisms evolved from unicellular life, previous achievements like tactic behavior had to be

\footnotetext{
* Correspondence: armin.hallmann@gmx.de

'Department of Cellular and Developmental Biology of Plants, University of Bielefeld, Universitätsstr. 25, 33615 Bielefeld, Germany
}

modified to fulfill the requirements of multicellularity [1-4]. The volvocine green algae, a group of species closely related to the "genus" Volvox within the order Volvocales (Chlorophyta), represent an excellent set of organisms for analyzing (photo)tactic responses during evolution of multicellularity [4-6]. This group of algae spans the full range of complexity from unicellular genera, such as Chlamydomonas, to colonial genera, such as Eudorina, to multicellular genera, such as Volvox [5]. 
Phylogenetic analyses indicate that multicellularity evolved much more recently in volvocine green algae than in any other group [7]. Phylogenetic analyses of the order Volvocales also revealed a polyphyletic origin of the "genus" Volvox [5,8-13]. A few species of this genus, including Volvox rousseletii, form a small but robust monophyletic group, which is called the section Volvox [10,12,14-16]. All other species of the genus Volvox (with the exclusion of the section Volvox) and the genera Eudorina and Pleodorina constitute another, much larger monophyletic group, the Eudorina group $[16,17]$ (see Additional File 1 for details).

All volvocine algae are flagellates that are dependent on phototactic behavior because they require sufficient light for photosynthesis and therefore need to orientate themselves in an efficient manner (for review, see [18]). For this, each (somatic) cell of each volvocine alga has two flagella for locomotion and an eyespot apparatus for light perception. The photoreceptors involved in the photoresponse have been identified both in Chlamydomonas reinhardtii [19-22] and Volvox carteri [23,24]. These photoreceptors are integral parts of a primordial visual system, forming, with the pigmented eyespot, a functional eye, which seems to share the same ultrastructure and physiology in both the unicellular Chlamydomonas and the multicellular Volvox [5,25-28].

In fact, the somatic cells of Volvox and its relatives are called "Chlamydomonas-like" in countless publications particularly due to the similarity of their eyes (and due to the presence of two flagella). Volvox, however, is much more than a multicellular version of Chlamydomonas. To allow for effective forward swimming and phototaxis, multicellular organization created two major requirements: (1) the appropriate arrangement and orientation of the biflagellated cells and (2) the coordination of cell behaviors.

Initially, a spherically symmetric arrangement and orientation of identical biflagellated cells might appear to be an ideal configuration for a multicellular organism. A hypothetical 5000-celled, spheroidal Chlamydomonas, however, would not be able to display any effective motility, not to mention phototaxis [29]. Forward swimming would be impossible because each cell in such a spherical surface monolayer would have a counterpart on the opposite side of the spheroid with flagella pointing in the opposite direction, and therefore the flagellar forces generated by opposing cells would neutralize each other. Because Volvox is actually a spheroidal organism, questions arise as to how it actually swims forward and what is different from the swimming motion of Chlamydomonas. Although Chlamydomonas and Volvox swim forward and rotate simultaneously, they have to do it in a very different manner owing to their different geometries and complexities. Chlamydomonas swims toward the cellular anterior with a breaststroke-like motion and rotates counterclockwise (as viewed from behind the cell) about the cellular anterior-posterior axis [30-35]. It switches between nearly straight-ahead swimming with synchronous beating and abrupt large reorientations with asynchronous flagellar beating, which has been described as a eukaryotic version of the "run-andtumble" motion of peritrichously flagellated bacteria [36]. Because the Chlamydomonas cell rolls around its longitudinal cell axis during helical forward swimming, its eye receives a sinusoidally modulated light signal. In addition to phototaxis, Chlamydomonas also shows photophobic responses $[34,35,37,38]$. The photophobic response is caused by a switch in the flagellar waveform, whereas phototaxis is caused by changing the balance of beating between the two flagella [39-41].

In the spheroidal colonial and multicellular volvocine species, the cellular anterior-posterior axis is different from the anterior-posterior axis of the whole organism. The effective strokes of both flagella beat in the same or almost the same direction toward the posterior pole of the spheroid, and they beat in parallel planes pushing the spheroid in the posterior-anterior direction [42-44]. This type of beating is indispensable for the effective forward swimming of spheroidal colonial and multicellular flagellates; it evolved together with the transition from unicellularity to multicellularity and is caused by the rotation of the basal bodies that underlie the flagella and determine their orientations $[6,8,42,45]$. The net effective propulsion generated by the effective strokes is not directed exactly parallel to the posterior-anterior axis of the spheroid and therefore results in rotation during forward swimming $[29,44,46]$; this characteristic forward rolling motion is also reflected in the name of the Volvox genus (Latin for "fierce roller") [47]. The arrangement, orientation, and beating direction of the biflagellated cells, however, explain only the reason that Volvox can swim forward but not its phototactic behavior.

For more than 100 years, scientists have studied the swimming behavior and phototaxis in multicellular (and colonial) volvocine algae (e.g., [29,46,48-61]). A key question in these studies on the phototactic behavior in multicellular volvocine algae was always how the coordination among their cells works. A cell-to-cell communication network among somatic cells that serves as the basis for the coordination of phototactic behavior seems to be rather obvious. Indeed, several species of the genus Volvox possess a network of cytoplasmic bridges even at the adult stage, which could allow for signal transduction between cells. However, amazingly, the multicellular volvocine algae are able to accomplish phototactic swimming without any known direct communication among cells $[49,62,63]$. This lack of 
communication becomes clear in species of the genus Volvox that lack any intercellular connections in adults (e.g., V. carteri); these species are well coordinated and swim toward the light, just as those with connections do. Likewise, individuals from species with cytoplasmic connections (e.g., $V$. globator) that had their connections microsurgically disrupted still showed phototactic behavior $[49,62,63]$. These observations bring up the question: How does the coordination of phototactic behavior work without direct communication among cells?

A change in the swimming direction in colonial and multicellular volvocine algae results from changes in the flagellar activity of cells in different portions of the spheroid. For $V$. aureus and V. carteri (both from the Eudorina group), fluid streamlines at the anterior pole were shown to stop transiently upon illumination, suggesting that cells at the anterior pole are responsible for the photoresponses [53-55,64]. Earlier, Fritsch [65] reported that cells at the anterior pole of the spheroid have larger eyespots than those at the posterior pole, which supported the idea of more sensitive eyes at the anterior pole $[29,55]$. Sakaguchi and colleagues $[54,55]$ concluded that minor changes in the behavior of cells at the anterior pole (of $V$. carteri and $V$. aureus) that come into or out of an illumination beam path during the spheroid's swimming with rotation cause both positive and negative phototaxis.

Previously, two competing models for positive phototactic steering in Volvox had been proposed. In the "variable beat frequency model" [48,52-55] the cells at the anterior pole reduce their beat frequency as the light intensity changes as cells rotate from the shaded side to the one in the light. In addition, they increase their beat frequency as they rotate from the illuminated side to the one in the dark. In the "variable beat direction model" [46], the cells change their flagellar beat direction when the cells rotate from the shaded side to the one in the light and also when they rotate from the illuminated side to the one in the dark. On the dark side, the flagella beat along the anterior-posterior axis toward the posterior pole of the spheroid, whereas on the illuminated side, the flagella beat laterally. Hoops et al. [57] tested both models in $V$. carteri, and their results supported the variable beat frequency model while being inconsistent with the variable beat direction model for phototactic turning.

A recent mathematical theory and measurements in $V$. carteri suggest that colony rotation and photoresponsive kinetics have evolved to be mutually tuned and optimized for phototaxis [61].

In the present study, the phototactic behavior in the spheroidal, multicellular volvocine green alga $V$. rousseletii (section Volvox), one of the fastest swimmers among all volvocine green algae, was analyzed to address the question of how a multicellular organism accomplishes phototactic swimming without any known direct communication among cells. To our knowledge, this is the first detailed description of phototactic behavior in a species within the section Volvox.

In response to light stimuli, not only was the beat frequency in cells on the anterior hemisphere changed, but also the beat direction was reversed. Moreover, the flagella waveform changed when the cells reversed the beat direction in response to changes in light intensity. The pulsed illumination of somatic cells at the anterior pole demonstrated that $V$. rousseletii quickly responds to changing light conditions with a reversal of the beating direction. We show that positive phototaxis is assisted by gravitational forces and that there is no negative phototaxis in $V$. rousseletii (under our experimental conditions). Finally, we present a model for positive phototactic steering in Volvox rousseletii that is consistent with both our results and those of previously published studies.

\section{Results}

\section{Phylogenetic analysis of Volvox rousseletii strain MI01}

The wild-type Volvox rousseletii strain MI01 was isolated in 1998 from the Machi-ike pond in Oh-gata, Tsukuba-shi, Ibaraki, Japan. A phylogenetic analysis of this strain was made to confirm its identity, place it accurately within the phylogenetic tree, and confirm that it belongs to the section Volvox. The analysis was based on cDNA fragments of chloroplast genes ( $p s a \mathrm{~A}, p s a \mathrm{~B}$ and $r b c \mathrm{~L}$ ) and an internal transcribed spacer sequence (ITS2) from 47 volvocine species [11,16,17,66-70]. The background and procedures of the phylogenetic analysis are described in Additional File 2. Detailed information for the cDNA sequences (e.g., source species and/or strains, abbreviations, accession numbers and references) is described in Additional File 3. The alignments that were performed are shown in Additional Files 4, 5 and 6 , and the sequence identity calculations are described in Additional Files 7, 8, 9 and 10. A consensus tree based on 30,000 resampled data sets for the psaA, psa $\mathrm{B}$ and $r b c \mathrm{~L}$ cDNA fragments $(10,000$ data sets from each) is shown in Additional File 1, together with some phylogenetic commentary. The phylogenetic tree indicates polyphyly of the genus Volvox, which is consistent with several previous studies $[10,12,16,17]$. The species $V$. barberi, $V$. globator and $V$. rousseletii appear on the same branch, which corresponds to the monophyletic section Volvox $[5,14,16,17]$. The species used in this study, Volvox rousseletii strain MI01, appears next to the Volvox rousseletii UTEX 1862 strain.

\section{Polarity of the $V$. rousseletii spheroid}

An adult $V$. rousseletii alga consists of a spherical surface monolayer of $\sim 5000$ small biflagellate, terminally 
differentiated somatic cells and 1-20 asexual reproductive cells (the number greatly depends on environmental conditions). The cells are regularly positioned in a transparent spheroid of a glycoprotein-rich extracellular matrix, and this spheroid displays a distinctive morphological polarity along its anterior-posterior axis. The whole organism view shows that the spheroid is somewhat more enlarged along the anterior-posterior axis than along the equatorial axis (Figure 1c). In embryogenesis, mature asexual reproductive cells, which are located in the posterior hemisphere, undergo embryonic cleavage divisions, with the characteristic cleavage pattern of the embryo establishing the polarity of the organism. The fully cleaved embryo is inside out and must turn right side out in a gastrulation-like process called inversion [5]. Next, the juvenile spheroids within the adult increase in size by depositing large quantities of extracellular matrix and finally hatch from their parent. The juvenile spheroids are always located within the posterior hemisphere due to the position of the original reproductive cells (Figure 1c). The juvenile spheroids cause the posterior hemisphere to be denser than the anterior hemisphere [71,72], with a likely contribution by the closer spacing of somatic cells on the posterior hemisphere (see next paragraph). This anisotropic mass distribution ensures that the spheroids always sink with their posterior pole first.

Only volvocine algae in the section Volvox, to which $V$. rousseletii belongs, retain robust cytoplasmic bridges even in the adult $[5,14]$. An extensive network of these characteristic cytoplasmic bridges makes the somatic cells appear stellate when viewed from their flagellar ends (Figs. 1a, 1 to 4, and 1b, 1 to 4 ) and bell-shaped when viewed from the side (shown schematically in Figs. $2 \mathrm{i}$ to $2 \mathrm{l}$ ). The somatic cells at the anterior pole are larger than those at the posterior pole, and the cell-tocell distances at the anterior pole are also larger (Figure 1, a1 and 1, b1). Owing to the transparent extracellular
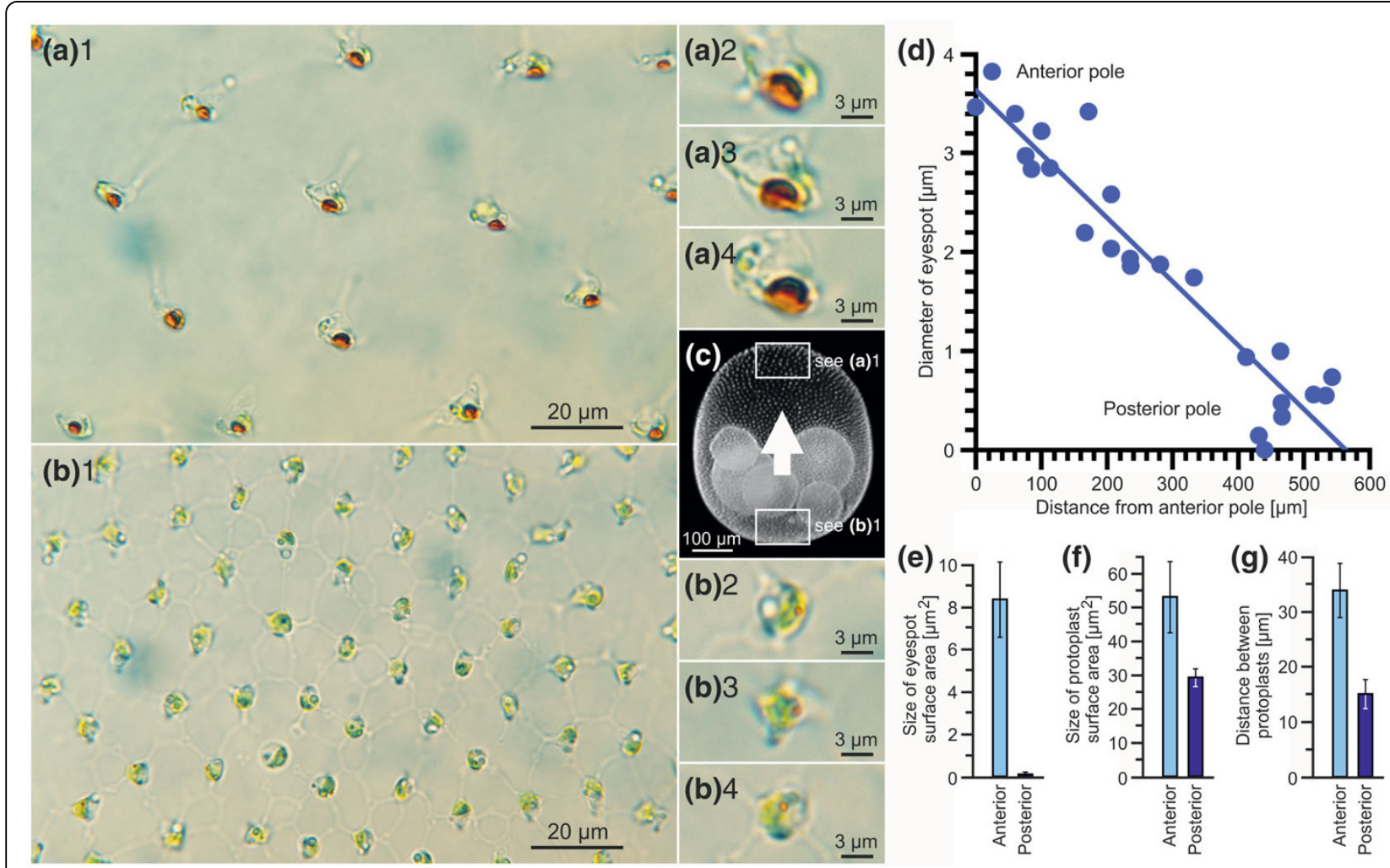

Figure 1 Organismal polarity of $\boldsymbol{V}$. rousseletii. (a)1 Closeup view of somatic cells at the anterior pole (see frame in Figure $1 \mathrm{c}$ ). (a)2-(a)4 Individual somatic cells from the anterior pole at high magnification. (b) 1, Closeup view of somatic cells at the posterior pole (see frame in Figure 1c). (b)2-(b)4 Individual somatic cells from the posterior pole at high magnification. (c) The swimming direction of $V$. rousseletii along its posterior-anterior axis is indicated by a white arrow. The progeny are within the posterior hemisphere. The polar diameter of the spheroid is always greater than the equatorial diameter. The frames indicate the areas shown in (a) 1 and (b)1. (d) The diameter of the eyespots was plotted against the linear distances between the respective somatic cells and the anterior pole $(n=24)$. (e) Size of the eyespot surface area at the anterior and posterior poles $(\mathrm{n}=15)$. (f) Size of the protoplast surface area at the anterior and posterior poles $(\mathrm{n}=60)$. (g) Distance between protoplasts at the anterior and posterior poles $(\mathbf{n}=250)$. (e)-(g) Error bars indicate the standard deviation of the $y$-value. $(\mathbf{d})$ - $(\mathbf{g})$ The sizes and distances were calculated from the photomicrographs. 

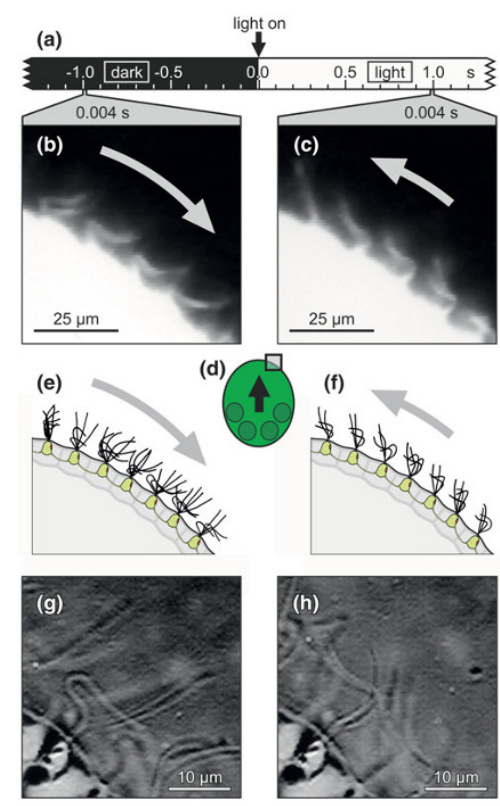

(f)
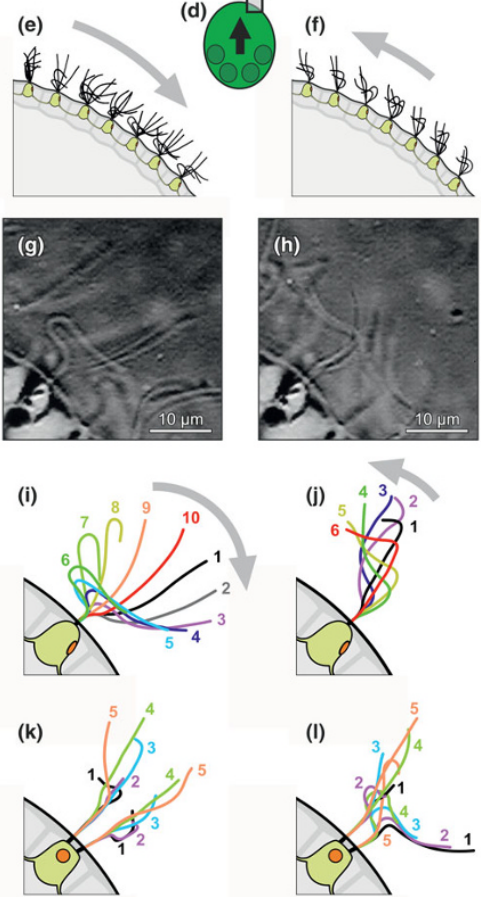

Figure 2 Flagellar strokes and flagellar waveforms of the normal and reverse beating mode. The flagellar activity of somatic cells before and after light stimulation. Light gray arrows indicate the direction of the effective flagellar strokes. (a) Time scale. (b) and (c) Dark-field images of somatic cells close to the anterior pole (see Figure 2d). Both images show the same cells (exposure time, $0.004 \mathrm{~s}$ ). (b) The flagellar activity before the light was turned on (normal beating mode). (c) The flagellar activity immediately (1.0 s) after the light was turned on (light intensity: $20.8 \mu \mathrm{mol} \mathrm{m} \mathrm{m}^{-2}$ ). The flagella showed the reverse beating mode. (d) The frame indicates the analyzed area relative to the spheroid, and the black arrow indicates the spheroid's inherent moving direction. (e) and (f) Stylized representation of the recorded flagellar strokes of somatic cells before and immediately (1.0 s) after light stimulation. (g) and (h) Frames from high-speed captured microvideographs of the same somatic cell. The viewing angle is almost perpendicular to the bending plane of the flagella. (g) Normal beating mode (before light stimulation). (h) Reverse beating mode immediately (0.1 s) after light stimulation. (i) and (j) Superimposed flagellar waveforms derived from successive stop-motion, high-speed video frames of the cell shown in (g) and (h) that show complete stroke. Only one flagellum is shown. (i) Normal beating mode. (j) Reverse beating mode. (k) and (I) Superimposed flagellar waveforms of both flagella of a somatic cell in normal beating mode. To allow for the simultaneous tracing of both flagella, the viewing angle is almost parallel to the bending plane of the flagella. The interval between the flagellar traces in $(\mathrm{k})$ and $(\mathrm{I})$ is only $0.13 \mathrm{~s}$. matrix, the outer edge of each somatic cell is indistinct; therefore, we measured the protoplast surface areas and distances between protoplasts. When compared with the posterior pole, the protoplast surface areas and the distances between protoplasts at the anterior pole were 1.8-fold larger (Figure 1f) and 2.3-fold longer (Figure $1 \mathrm{~g})$, respectively. The decrease in cell distances along the anterior-posterior axis also becomes apparent in the view of the whole spheroid (Figure 1c), and as mentioned above, this closer spacing of somatic cells on the posterior hemisphere might contribute to the higher density of the posterior hemisphere. The somatic cells at the anterior pole have large eyespots $(\sim 3.6-\mu \mathrm{m}$ diameter) that face toward the posterior pole (Figure 1a, 1 to 4). The diameter of the eyespots decreased continuously along the anterior-posterior axis (Figure 1d). Cells at the posterior pole have either a tiny or an undetectable eyespot. Those eyespots that are present do not face in the same direction (Figure 1b, 1 to 4 ). A comparison of the eyespot surface areas of cells at the anterior pole with those at the posterior pole highlights these differences in eyespot size; the surface areas at the anterior pole are $\sim 80$-fold larger than those at the posterior pole (Figure 1e), indicating that the cells at the anterior pole possess the highest light sensitivity and that the sensitivity decreases continuously along the anterior-posterior axis toward the posterior pole. The cells at the posterior pole are light-insensitive and essentially blind.

\section{Efficiency of phototactic movements as a function of light intensity}

The phototactic movements of the $V$. rousseletii spheroids were analyzed in vertical (setup A, Figure 3a) and horizontal directions (setup B, Figure 3b) under continuous unidirectional illumination with a broad range of intensities spanning approximately eight orders of magnitude (Figure 3c). For the calculation of the photoaccumulation index (PI), the observation chamber was divided into six (setup A) or three (setup B) areas, with area 1 closest to the light source.

An example of the calculation of the PI for all areas at $0.16 \mu \mathrm{mol} \mathrm{m}{ }^{-2} \mathrm{~s}^{-1}$ in setup A is shown in Figure 3d. When the light is on, the PI for area 1 remains relatively constant for $\sim 5 \mathrm{~s}$ and then increases continuously. The initial delay is caused by a reduction in swimming speed after the dark-light switch (see below sections "Swimming speed after a dark-light switch as a function of light intensity" and "Swimming speed after light stimulation as a function of the swimming direction"). The PI for area 1 reaches its maximum after $\sim 25 \mathrm{~s}$, whereas the PI of the other areas decreases in return. The PI for area 2 increased temporarily because the spheroids have to cross this area to reach area 1 . In Figures $3 \mathrm{e}$ to $3 \mathrm{~h}$, 
(a)

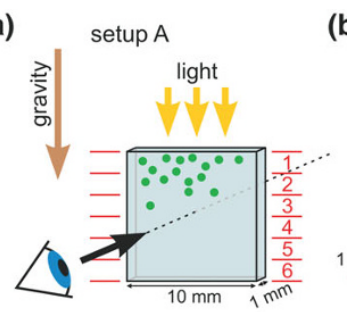

(c)

(C) $\operatorname{setup} A$

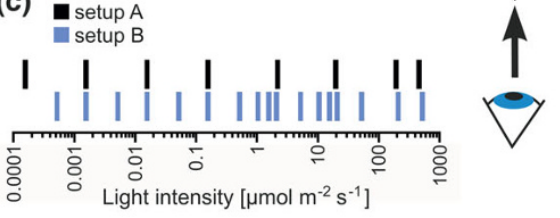

(e)

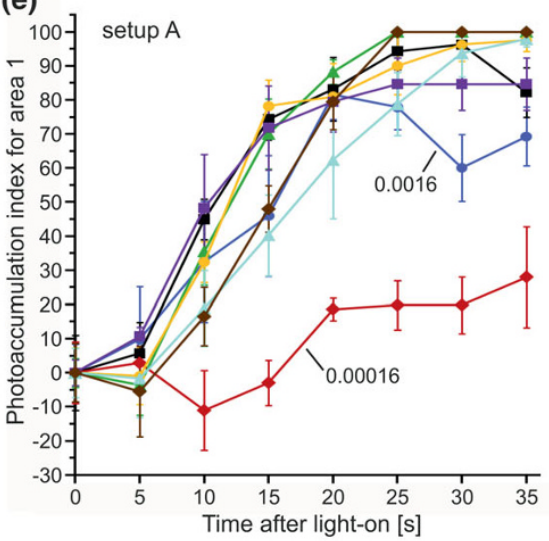

(g)

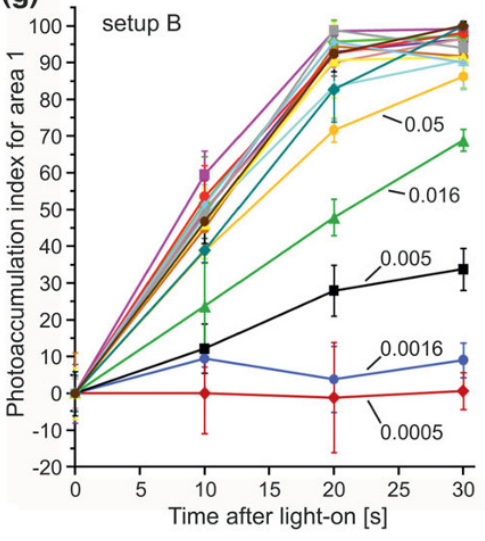

(b) $\operatorname{setup~B~}$

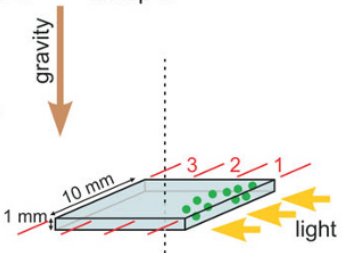

(d)

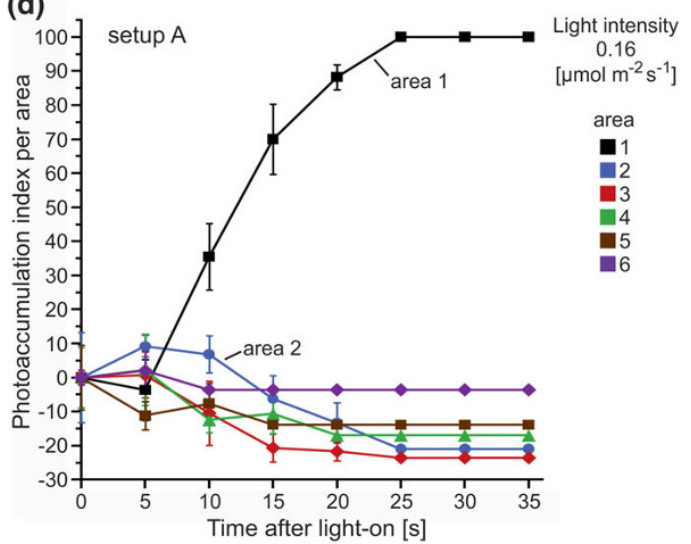

(f) Light intensity $\left[\mu \mathrm{mol} \mathrm{m} \mathrm{m}^{-2} \mathrm{~s}^{-1}\right]$ - 457 457
-191 $\square 19.6$ 2.2
$\square 0.16$ 0.16
$\square 0.016$ 0.0016 0.00016

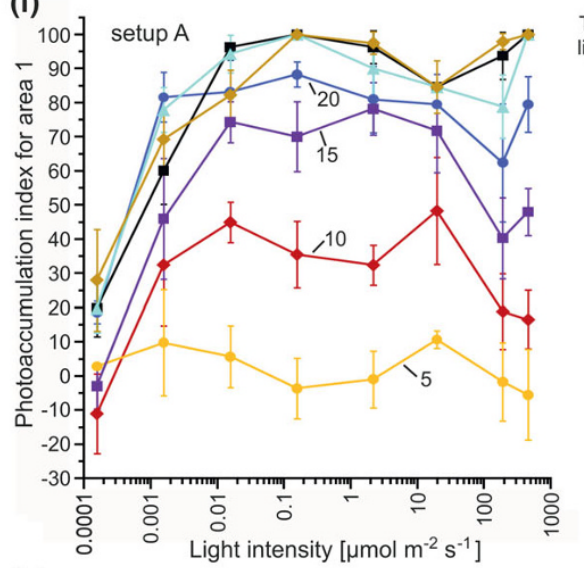

Time after light-on [s]

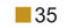

$\mathbf{r}$
$\mathbf{\square}$
$\mathbf{2}$
$\mathbf{2}$
$\mathbf{0}$
$\mathbf{2}$

$\square+20$

15
$\square 10$

5

h)

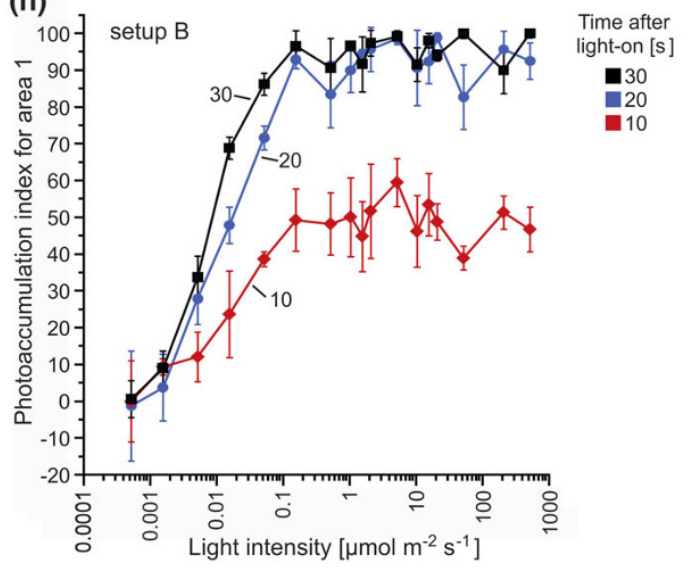

Figure 3 Quantification of the phototactic movements depending on the applied light intensity. (a) and (b) The viewing direction, the direction of the light vector and the direction of the gravity vector is indicated. (a) In the vertical setup A, the direction of the light vector is identical to the direction of the gravity vector. The observation chamber was divided into six areas with area 1 being closest to the light source. (b) In the horizontal setup B, the direction of the light vector is perpendicular to the direction of the gravity vector. The observation chamber was divided into three areas with area 1 being closest to the light source. (c) Schematic representation of the light intensities used in setups $\mathrm{A}$ and B. (d)-(h) Phototactic movements were quantified by calculating the photoaccumulation index (PI). The higher the PI value, the stronger the photoaccumulation. (d) The PI was calculated separately for each area (setup A) and was plotted against the time after the light was turned on (light intensity: $0.16 \mu \mathrm{mol} \mathrm{m} \mathrm{m}^{-2} \mathrm{~s}^{-1}$ ). (e) The PI for area 1 in setup A was plotted against the time after the light was turned on using eight different light intensities. (f) The PI for area 1 in setup A was plotted against the different light intensities. Seven different times were analyzed separately at intervals of $5 \mathrm{~s}$. (g) The PI for area 1 in setup B was plotted against the time after the light was turned on using 17 light intensities. (h) The PI for area 1 in setup B was plotted against the different light intensities. Three different times were analyzed separately at intervals of 10 s. (d)-(h) The color assignment is indicated. Error bars refer to the standard deviation of the $y$-value $(n=3)$. 
only the results for area 1 are shown because they sufficiently describe the photoaccumulation.

In the vertical setup A, light intensities of $0.016 \mu \mathrm{mol}$ $\mathrm{m}^{-2} \mathrm{~s}^{-1}$ or above resulted in the same phototactic behavior as described above (Figure 3e and 3f). Remarkably, high light intensities of up to $457 \mu \mathrm{mol} \mathrm{m} \mathrm{m}^{-2} \mathrm{~s}^{-1}$ also yielded strong positive phototaxis, with the spheroids accumulating in area 1 within $\sim 25 \mathrm{~s}$. Even very low light intensities $\left(0.00016 \mu \mathrm{mol} \mathrm{m}^{-2} \mathrm{~s}^{-1}\right)$ resulted in a small increase of the PI. The photoaccumulation in the horizontal setup B (Figure $3 \mathrm{~g}$ and $3 \mathrm{~h}$ ) was essentially the same as in the vertical setup. In contrast, very low light intensities (i.e., 0.0016 $\mu \mathrm{mol} \mathrm{m} \mathrm{m}^{-2}$ or below) had no significant effect. The lower sensitivity at low light intensities in setup B compared with setup A seemed to be caused by the facilitating effects of gravity on phototaxis (see sections "Swimming speed after light stimulation as a function of the swimming direction" and "Gravity facilitates positive phototaxis" in the Discussion), which might not be observed in setup B due to the dimensions of the observation chamber combined with the direction of the light vector. At light intensities between 0.005 and $0.16 \mu \mathrm{mol} \mathrm{m}^{-2} \mathrm{~s}^{-1}$, the PIs at 10,20 , or $30 \mathrm{~s}$ after the light is switched on increase with increasing light intensity (Figure 3h), such that the higher the intensity, the earlier the spheroids reach the light source. There is no further increase in PI at intensities above $0.16 \mu \mathrm{mol}$ $\mathrm{m}^{-2} \mathrm{~s}^{-1}$ (Figure 3h). As in setup A, even the high light intensities (up to $520 \mu \mathrm{mol} \mathrm{m}^{-2} \mathrm{~s}^{-1}$ ) produce a strong positive phototaxis. These results indicate that there is no negative phototaxis in $V$. rousseletii (under the conditions of this study).

\section{Efficiency of phototactic movements after a longer dark period}

To determine whether the length of the dark period before the light stimulus influences the phototactic movements, light-adapted spheroids were kept in the dark for 0 to $90 \mathrm{~min}$ and subsequently subjected to a photoaccumulation assay (Table 1 ). These results demonstrated that longer dark periods reduced the efficiency of the phototactic movements, decreasing continuously from $100 \%$ to $~ 65 \%$ within an hour in the dark. Periods of darkness longer than 1 hour, however, did not result in any further decrease of significance.

\section{Swimming speed after a dark-light switch as a function of light intensity}

To determine the swimming speed of individual spheroids after a dark-light switch, the tracks of the spheroids were recorded as shown in Figure 4a. The speed of individual spheroids was determined at 1 -s intervals from $1 \mathrm{~s}$ before to $4 \mathrm{~s}$ after the dark-light switch. Eight different light intensities, spanning approximately eight orders of magnitude, were analyzed separately, and the relative swimming
Table 1 Reduced efficiency of the phototactic movements after a longer dark period

\begin{tabular}{ccc}
\hline $\begin{array}{c}\text { Period of Darkness Before Illumination } \\
\text { (min) }\end{array}$ & $\begin{array}{c}\text { Spheroids in Area } \mathbf{1}^{*} \\
(\mathbf{\%})\end{array}$ & $\begin{array}{c}\text { SD } \\
(\%)\end{array}$ \\
\hline 0 & 100 & 2.5 \\
$0-10$ & 97.3 & 3.1 \\
$10-20$ & 86.5 & 8.7 \\
$20-30$ & 87.7 & 3.3 \\
$30-40$ & 84.5 & 1.3 \\
$40-50$ & 81.5 & 4.1 \\
$50-60$ & 67.2 & 11.7 \\
$60-70$ & 65.2 & 6.1 \\
$70-80$ & 63.8 & 11.1 \\
$80-90$ & 61.9 & 13.6 \\
\hline
\end{tabular}

*The duration of the photoaccumulation assay was $30 \mathrm{~s}(\mathrm{n}=3$ for each time period). The number of light-adapted spheroids accumulated in area 1 (setup $\mathrm{B}$, Figure $3 \mathrm{~b}$ ) was set to $100 \%$. These light-adapted spheroids correspond to 0 $\mathrm{min}$ in the dark. The light intensity during both the light adaptation and the photoaccumulation assay was $2.08 \mu \mathrm{mol} \mathrm{m} \mathrm{m}^{-2} \mathrm{~s}^{-1}$.

speed of the spheroids was plotted against the time after the light was turned on (Figure 4b). A dark-light switch using low light intensities (i.e., between 0.00016 and 0.016 $\mu \mathrm{mol} \mathrm{m} \mathrm{s}^{-1}$ ) caused a speed reduction to $\sim 70 \%$ relative to the swimming speed immediately before light stimulation. Higher light intensities (i.e., between 0.16 and $457 \mu \mathrm{mol}$ $\mathrm{m}^{-2} \mathrm{~s}^{-1}$ ) caused a speed reduction to $35 \%$ to $45 \%$ (Figure $4 \mathrm{~b})$. These speed reductions occurred within $1 \mathrm{~s}$ of the onset of illumination, and the former speed before the onset of darkness was recovered in $\sim 3$ to $4 \mathrm{~s}$. This temporary speed reduction reflects a brief photophobic response of the organism. Overall, the results with all intensities indicate that the higher the light intensity, the stronger the speed reduction.

The absolute swimming speed of recently hatched spheroids in the dark (setup A, Figure 3a) was determined to be $\sim 880 \mu \mathrm{m} \mathrm{s}^{-1}$ (SD $270 \mu \mathrm{m} \mathrm{s}^{-1}, \mathrm{n}=50$ ) in an upward direction and $1060 \mu \mathrm{m} \mathrm{s}^{-1}$ (SD $440 \mu \mathrm{m} \mathrm{s}^{-1}, \mathrm{n}=$ $50)$ in a horizontal direction. In contrast, Solari and coworkers $[73,74]$ reported an upward swimming speed of $\sim 500 \mu \mathrm{m} \mathrm{s}^{-1}$ (up to $\sim 590 \mu \mathrm{m} \mathrm{s}^{-1}$ ) for $V$. rousseletii. These differences may be due to different culture conditions and a different experimental setup, particularly with respect to the dimensions of the observation chamber. In our experiments, the spheroids were only able to swim along the $x$ - and $y$-axes but not the $z$-axis, which extended only $0.1 \mathrm{~cm}$. Solari et al. used a chamber that extended $1 \mathrm{~cm}$ along the $z$-axis, allowing movements along this axis that may account for the lower values for the upward swimming speed ( $y$-axis).

\section{Swimming speed after light stimulation as a function of the swimming direction}

To investigate the swimming speed of individual spheroids after a dark-light switch relative to the swimming 
(a)

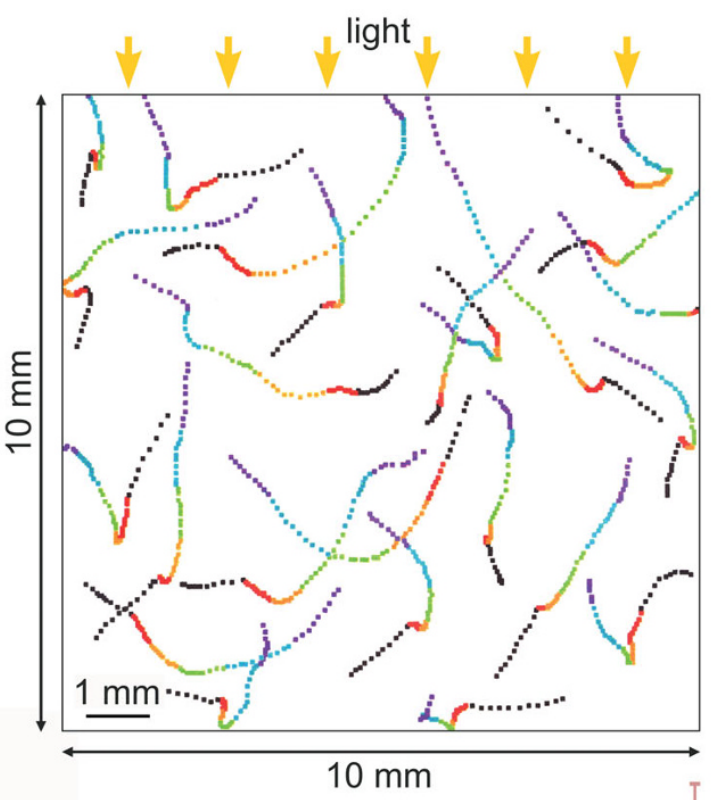

(b)

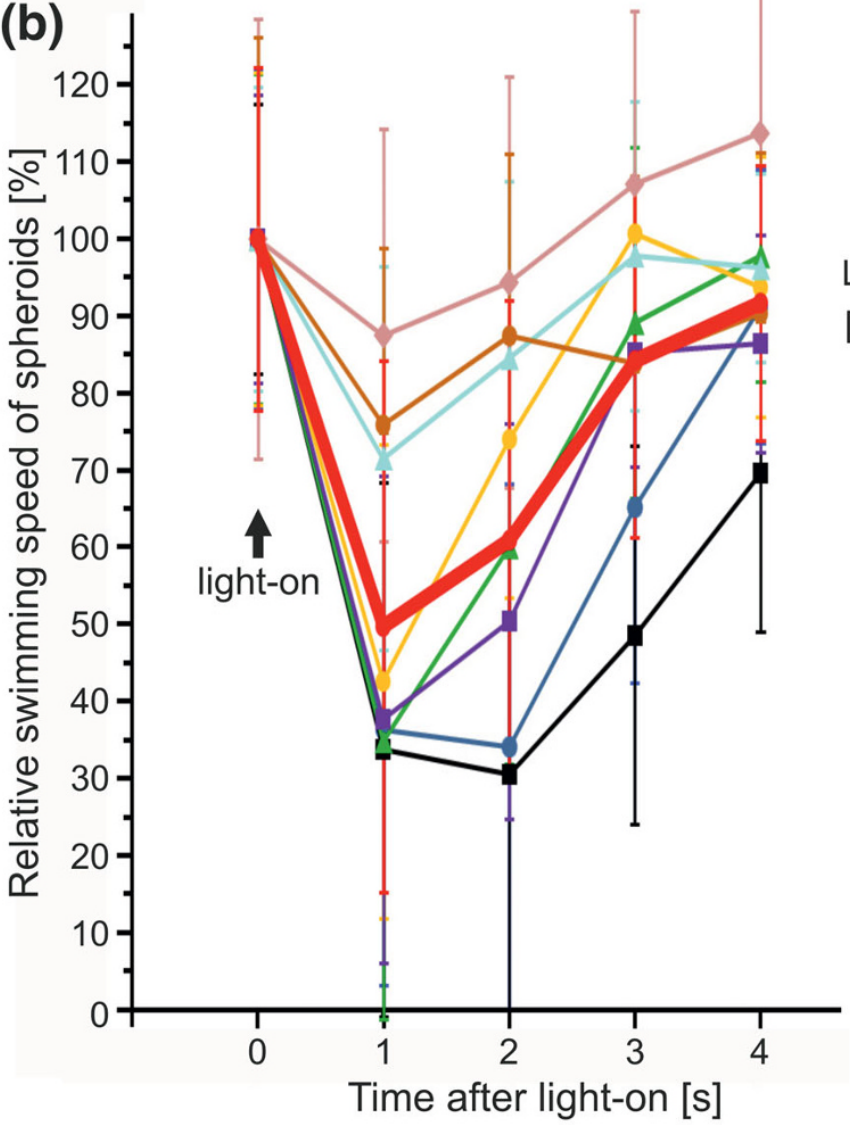

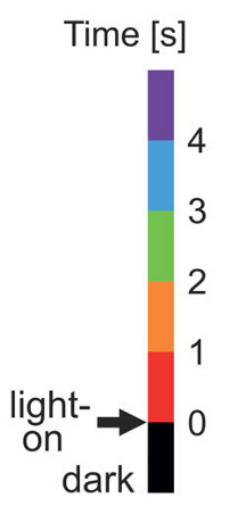

Light intensity $\left[\mu \mathrm{mol} \mathrm{m} \mathrm{m}^{-2} \mathrm{~s}^{-1}\right]$

457

191

19.6

2.2

$\square .16$

0.016

0.0016

0.00016

Figure 4 Swimming speed of $\boldsymbol{V}$. rousseletii spheroids after a dark-light switch, depending on the light intensity. (a) An example of the tracks of the spheroids in the observation chamber (vertical setup A, Figure 3a). The position of each spheroid was recorded every $0.1 \mathrm{~s}$ starting at $1 \mathrm{~s}$ before the dark-light switch. The color of the position marks changed every second as indicated. The yellow arrows indicate the light source (unidirectional illumination). (b) The relative swimming speed of spheroids was plotted against the time after the light was turned on (vertical setup A, Figure 3a). The swimming speed immediately before light stimulation was set to $100 \%$. A total of eight different light intensities were analyzed separately, and the color assignment is indicated. In addition, the average values for all intensities are given (bold red line). Error bars refer to the standard deviation of the $y$-value $(n=20)$. 
direction just before the light $\left(2.1 \mu \mathrm{mol} \mathrm{m} \mathrm{m}^{-2} \mathrm{~s}^{-1}\right)$ was turned on, setup A (Figure 3a) was used, and the spheroids were tracked as described above (Figure 4a). In each track, a line between the spheroid's position $1 \mathrm{~s}$ before the dark-light switch and its position right at the dark-light switch was drawn. The angle between each of these lines and the direction of the light vector was determined, and the angles were assigned to sectors a-i (see legend in Figure 5a). The speed of each spheroid was determined at 1-s intervals from $1 \mathrm{~s}$ before to $4 \mathrm{~s}$ after the onset of illumination. For each swimming direction in the dark (a-i), the relative swimming speed of the spheroids was plotted against the time after the onset of illumination (Figure 5a). The dark-light switch caused a speed reduction, regardless of the directions in which the spheroids were swimming immediately before the dark-light switch. This result is even true for direction a, in which the spheroids had already swum to the light source by chance as the light was turned on. There were, however, sector-dependent differences in speed reduction. For sectors a-c, speed was reduced to $\sim 30 \%$ to $45 \%$ compared with the swimming speed immediately before light stimulation, and for sectors $\mathrm{d}$ to i, it was reduced to $\sim 50 \%$ to $60 \%$ (Figure $5 \mathrm{a}$ ). These sectordependent differences become even clearer when the absolute swimming speeds are represented in a sectorwise manner (Figure 5b). There is already a sector dependency of the absolute swimming speed before the onset of illumination ( $0 \mathrm{~s}$, Figure $5 \mathrm{~b}$ ), indicating that spheroids swim $\sim 1.5$ times faster to the bottom than to the top. This sector-dependency remained when the light was switched on, and the absolute speed was promptly reduced ( $1 \mathrm{~s}$, Figure $5 \mathrm{~b}$ ). Interestingly, the sector dependency persists when the spheroids accelerate to their previous speed (2-4 s, Figure $5 b$ ). Consequently, the deceleration and acceleration of spheroids after a dark-light switch is independent from the swimming direction relative to the light source immediately before the onset of illumination, but it is influenced by gravitational force.

The analysis of the course of the tracks also made the impact of gravity apparent. Tracks from vertical setup A (Figure 3a) showed that the immediate speed reduction after the dark-light switch was accompanied by a change in the moving direction toward the bottom (Figure $5 \mathrm{c}$ ). This sinking takes $\sim 2 \mathrm{~s}$, and the spheroids drop by $\sim 0.45 \mathrm{~mm}$ (SD $0.15 \mathrm{~mm}, \mathrm{n}=20$ ) before they accelerate and swim to the light source. This temporary sinking is indicative of the brief photophobic response of the organism after the dark-light switch. Tracks from horizontal setup B (Figure $3 \mathrm{~b}$ ) also show an immediate speed reduction after the dark-light switch with subsequent acceleration as the spheroids swim to the light source (Figure 5d). Owing to the dimensions of the observation chamber $(0.1 \mathrm{~cm}$ in the vertical direction $)$ and the viewing direction (antiparallel to the gravitational force), the sinking characteristic is not seen in the horizontal setup B (Figure $5 \mathrm{~d}$ ).

\section{Fluid streamlines before and after a dark-light switch}

Visualization of fluid streamlines in a suspension of polystyrene beads [53] allowed the analysis of flagellar activity of $V$. rousseletii spheroids before and after a dark-light switch (Figure 6). In the dark, organisms that were gently trapped between a cover glass and a glass slide produced fluid streamlines in the anterior-posterior direction along the surface of the spheroid (Figures 6a and $6 \mathrm{~d}$ ). These streams resulted in forward swimming along the posterior-anterior axis in free-swimming spheroids. Exposure of the spheroids to illumination (2.1 $\mu \mathrm{mol} \mathrm{m} \mathrm{m}^{-2} \mathrm{~s}^{-1}$ ) caused a transient stop of the streams in front of the spheroid and a reversal of the fluid streamlines along the anterior hemisphere. In contrast, the fluid streamlines along the posterior hemisphere showed no significant change (Figure 6b). The particle velocities along the anterior hemisphere were lower in the reversed direction (Figure 6e) than in the anteriorposterior direction in the dark (Figure 6d). The reversal of the fluid streamlines indicates a reversed beating direction of flagella because the streamline pattern cannot be explained by a simple stop of flagellar beating as has been observed in $V$. carteri $[54,55]$. The reversal of the fluid streamlines lasts for 2 to 3 seconds immediately after the dark-light switch. The initial anterior-posterior fluid streamlines are then reestablished.

These results are consistent with the speed reduction and sinking of the spheroids after a dark-light switch described above and support the existence of a photoresponsive gradient from the anterior to the posterior pole of the spheroid, with the highest responses at the anterior and no response at the posterior.

\section{Flagellar strokes and flagellar waveforms before and after light stimulation}

Direct observation of flagellar activity by dark-field microscopy allowed an analysis of the cellular basis of the reversal of the fluid streamlines (Figures 2a-f). The flagellar activity was recorded $1 \mathrm{~s}$ before and $1 \mathrm{~s}$ after light stimulation with an exposure time of $0.004 \mathrm{~s}$ (Figure 2a). In the dark, the flagella of all somatic cells beat with a ciliary-type, asymmetrical motion, which we refer to as the normal beating mode (Figures $2 \mathrm{~b}$ and $2 \mathrm{e}$ ). This mode consists of an effective stroke in the anteriorposterior direction and a recovery stroke in the opposite direction. After the light stimulus, the cells within the anterior hemisphere completely reverse the flagellar beating direction, which we refer to as the reverse beating mode (Figures $2 \mathrm{c}$ and $2 \mathrm{f}$ ). Though the effective 


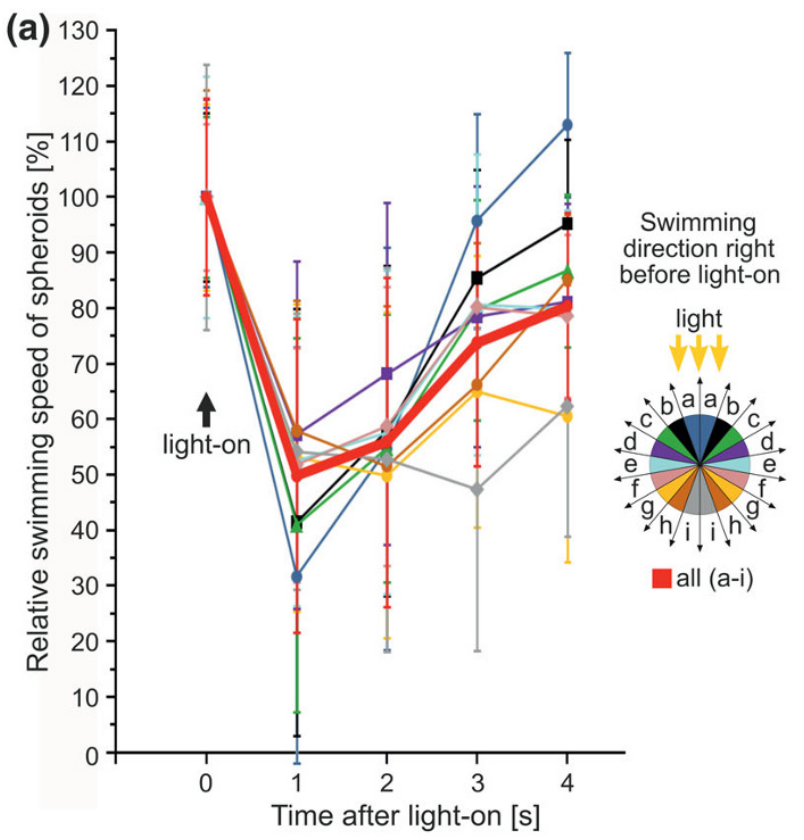

(b)

Absolute swimming speed of spheroids per swimming direction right before light-on

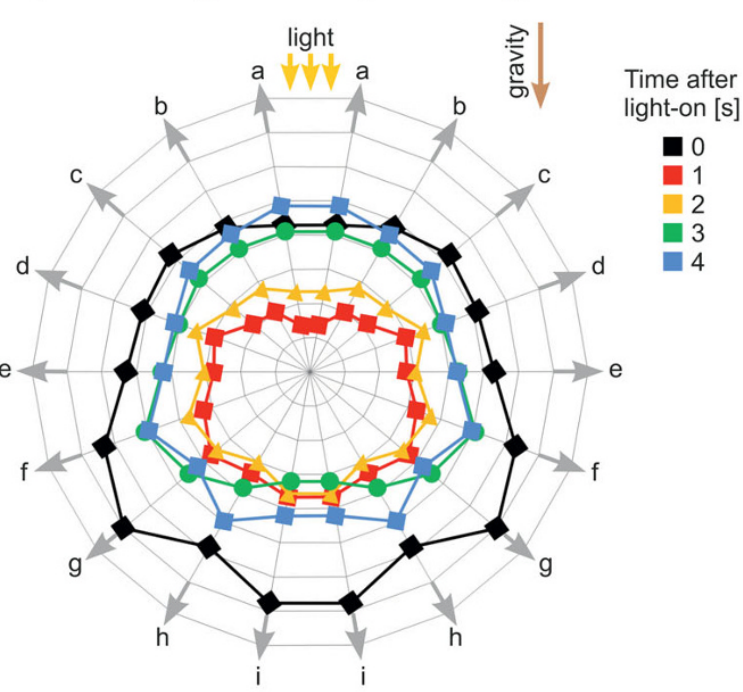

(c)

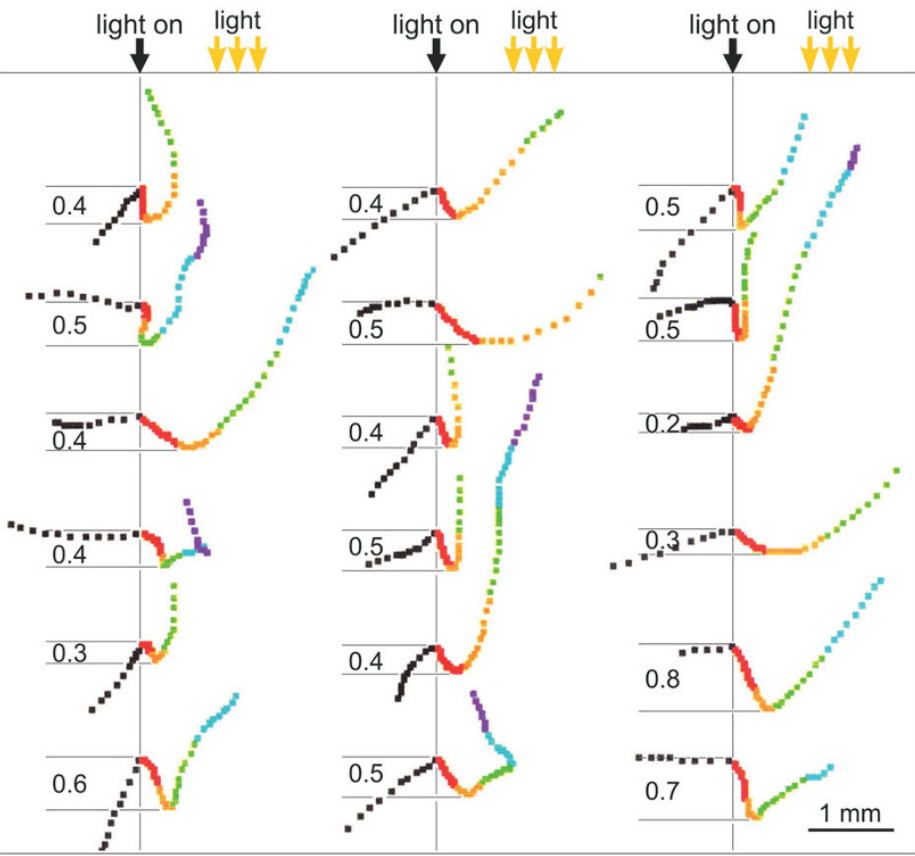

(d) light on light
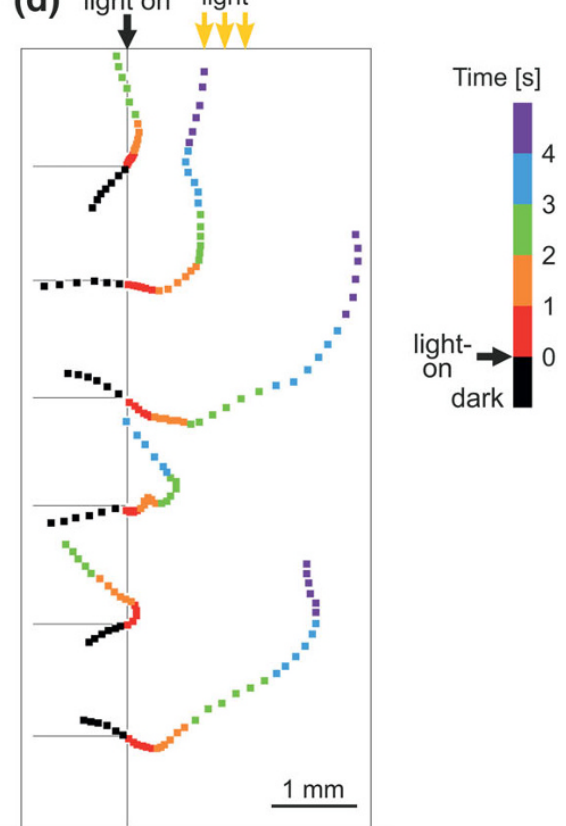

Figure 5 Swimming speed after a dark-light switch, depending on the swimming direction. (a) The relative swimming speed of the spheroids was plotted against the time after the light was turned on (vertical setup A). The swimming speed immediately before light stimulation was set to $100 \%$. The swimming direction relative to the light source immediately before light stimulation was recorded and assigned to different sectors (a-i), and the color assignment is indicated. Each sector was analyzed separately. In addition, the average values for all directions are given (bold red line). Error bars refer to the standard deviation of the $y$-value $(n=20)$. (b) The swimming direction relative to the light source immediately before light stimulation was recorded and assigned to different sectors (gray arrows, a-i). The absolute swimming speed of the spheroids is shown for 0-4 s after the light was turned on (vertical setup A). All swimming speeds were plotted against the swimming direction immediately before light stimulation. The color assignment, the direction of the light vector, and the direction of the gravity vector are indicated. Reading from the inside outward, the gray concentric circles indicate $0.2,0.4,0.6,0.8,1.0,1.2,1.4$, and $1.6 \mathrm{~mm} \mathrm{~s}^{-1}$. (c) and (d) The typical tracks of spheroids after a dark-light switch. The tracks were aligned relative to the moment of the dark-light switch (vertical lines), and the indicated color assignment reflects the time before and after the dark-light switch. (c) Tracks from vertical setup A, with the sinking depth of spheroids right after the dark-light switch indicated (in $\mathrm{mm}$ ). (d) Tracks from horizontal setup B. (a)-(d) The yellow arrows indicate the light source. The light intensity was $2.1 \mu \mathrm{mol} \mathrm{m} \mathrm{m}^{-1}$ (intensities up to $457 \mu \mathrm{mol} \mathrm{m} \mathrm{s}^{-1}$ produced the same results). 

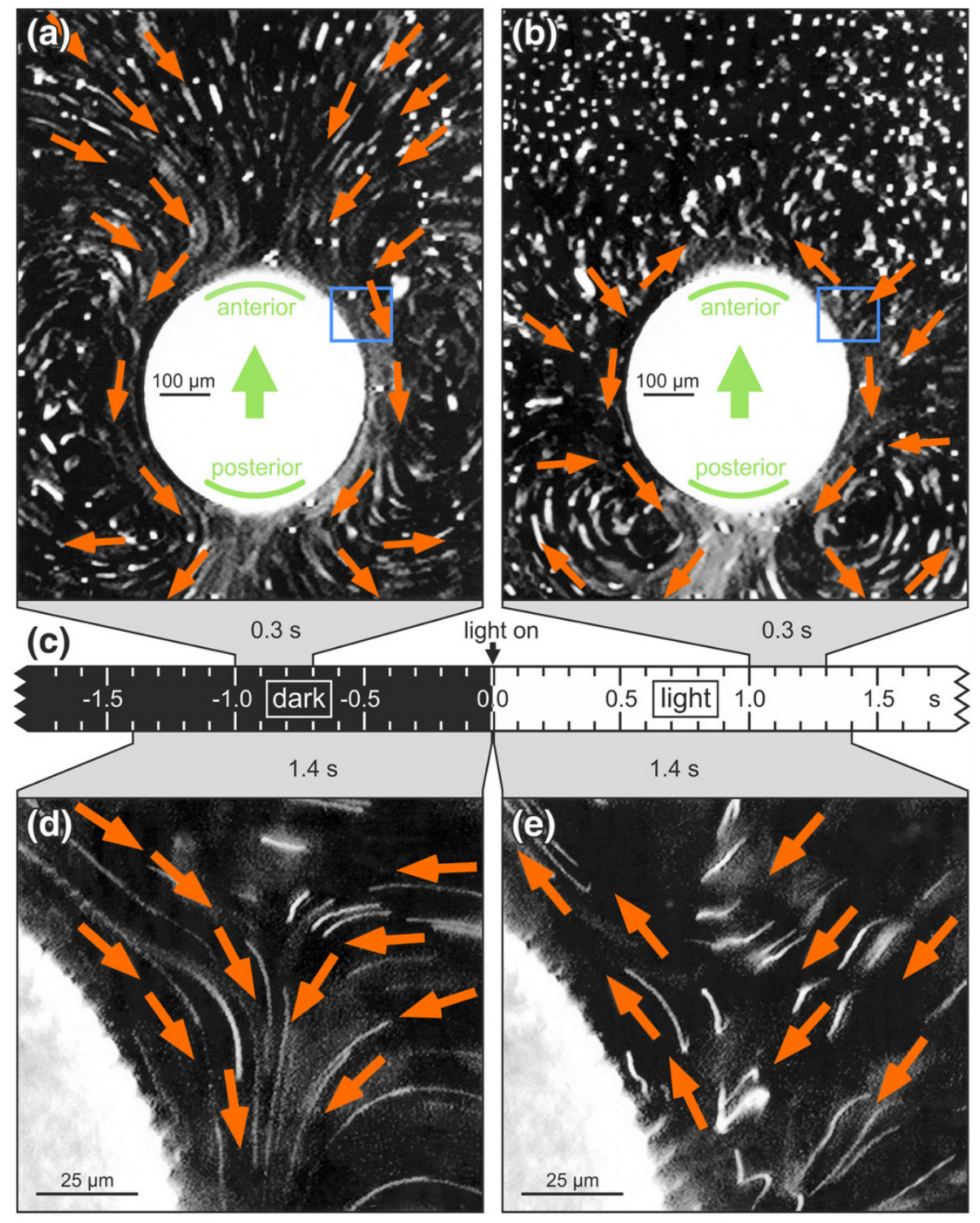

Figure 6 Fluid streamlines around a $\boldsymbol{V}$. rousseletii spheroid before and after light stimulation. The fluid streamlines using polystyrene beads were visualized and taken with long exposure times. (a) An exposure time of $0.3 \mathrm{~s}$ at 1 to $0.7 \mathrm{~s}$ before the light was turned on. (b) An exposure time of $0.3 \mathrm{~s}$ at 1 to $1.3 \mathrm{~s}$ after the light was turned on. (c) Time scale. (d) Closeup of a region near the anterior pole (see blue frame in a). An exposure time of $1.4 \mathrm{~s}$ at 1.4 to $0 \mathrm{~s}$ before the light was turned on. (e) Closeup of a region near the anterior pole (see blue frame in b). An exposure time of $1.4 \mathrm{~s}$ at 0 to $1.4 \mathrm{~s}$ after the light was turned on. Red arrows highlight the main streamlines, and green arrows in (a) and (b) indicate the inherent moving direction of the spheroid along the posterior-anterior axis. Light stimulation was performed with green light (2.1 $\left.\mu \mathrm{mol} \mathrm{m} \mathrm{m}^{-2}\right)$. The infrared light used for dark-field micrography was inactive for phototaxis. (a), (b), (d) and (e) show the same spheroid.

stroke is then in the posterior to anterior direction, the cells still beat with a ciliary-type motion. The reverse beating mode of cells on the anterior hemisphere lasts for 2 to 3 seconds, after which the cells switch back to the normal beating mode with the flagellar strokes and waveforms before light stimulation are completely identical to those 2 to 3 seconds after light stimulation. This result is consistent with the observed fluid streamlines after a dark-light switch as described above. The temporary reversal of the beating direction reflects a brief photophobic response at the cellular level.

High-speed microvideography was used to examine the flagellar strokes and waveforms of individual somatic cells more closely. Frames from the captured high-speed videos of a somatic cell on the anterior hemisphere show the normal beating mode (in the dark; Figure $2 \mathrm{~g}$ ) and the reverse beating mode $(0.1 \mathrm{~s}$ after light stimulation; Figure $2 \mathrm{~h}$ ). The superimposed flagellar waveforms 
show the complete stroke cycles of one flagellum in the normal (Figure 2i) and the reverse (Figure $2 \mathrm{j}$ ) beating modes. The reverse beating mode is only transient, with the somatic cells returning to their normal beating mode after adaptation to the light, which occurs after approximately 2 to 3 seconds. Cells in the normal beating mode show a typical base-to-tip, ciliary-type motion. The stroke area has a wide spread, and the tip of the flagellum describes a relatively wide arc during its effective stroke (Figure 2i). One stroke takes $\sim 45 \mathrm{~ms}$ (frequency $22 \mathrm{~Hz}$ [SD $6 \mathrm{~Hz}, \mathrm{n}=15]$ ) in the normal beating mode. In the reverse beating mode, one stroke takes $\sim 25 \mathrm{~ms}$ (frequency $40 \mathrm{~Hz}$ [SD $9 \mathrm{~Hz}, \mathrm{n}=15]$ ), and the cells also show a ciliary-type motion. There is, however, less bending close to the base of the flagellum and a much smaller stroke area, such that the tip of the flagellum describes only a small arc during its effective stroke (Figure 2j).

The description of flagellar waveforms requires that the angle of view be essentially perpendicular to the bending plane of the flagella. From this angle of view, only the beat of one flagellum, the one closest to the observer's eye, can be described properly. To observe both flagella of a cell at the same time, the angle of view was changed so that it was almost parallel to the bending plane of the flagella (Figures $2 \mathrm{k}$ and $2 \mathrm{l}$ ). The beating direction of the two flagella of a somatic cell is roughly parallel, and the effective strokes are generally directed toward the posterior pole. There is no evidence for any spatial or temporal coordination between the two flagellar beat cycles of a somatic cell, which is indicative of the asymmetrical, ciliary-type beating or between the beat cycles of the neighboring somatic cells. An example of the strokes of a single somatic cell in the normal beating mode is shown in Figures $2 \mathrm{k}$ and $2 \mathrm{l}$. The strokes of the two flagella shown in Figure 21 were recorded only $0.13 \mathrm{~s}$ after the strokes in Figure $2 \mathrm{k}$ without any changes in light or other conditions. A comparison of these strokes not only illustrates the asynchronous and asymmetrical beating of the flagella but also documents the three-dimensional component of the strokes. Beat cycles that were somewhat out of the normal bending plane were repeatedly observed, which may be a matter of slightly variable fluid resistance due to microturbulences caused by flagella from other cells.

\section{Simulation of phototactic swimming with a periodically changing light intensity due to the rotation of the spheroid}

A Volvox rousseletii spheroid always swims along its posterior-anterior axis while rotating about its posterioranterior axis in a counterclockwise direction (as viewed from behind the spheroid). Spheroids make one complete counterclockwise rotation approximately every 1 to
3 seconds (Figure 7a), with smaller (younger) spheroids rotating faster than larger (older) spheroids. The eyespots of somatic cells are highly directional optical devices that guide the organism to places where the light conditions are optimal for photosynthetic growth. The light signals change periodically during phototactic swimming due to the rotation of the spheroid and the directivity of the eyespot with its layers of carotenoidfilled granules below the photoreceptors in the plasma membrane [28] (Figure 7c). The periodically changing light signals of a rotating spheroid during phototaxis were simulated using a rotating filter system (Figure $7 \mathrm{~b}$ ), and fluid streamlines from three regions at the anterior pole were analyzed (Figure 7a). The construction of the rotating filter system was based on previous results on the directivity of photoreception in the unicellular alga Chlamydomonas [75] (Figures 7d and 7e), which corresponds to the situation in somatic cells on the anterior hemisphere of a Volvox spheroid (Figure 7d, blue line) as viewed from the flagellar end of the cells (Figure $7 d$, inset). The filter combinations and cutting of the filters were calculated to match the plot of the tangential electric energy density [75] at the plasma membrane overlaying the carotenoid-filled granules of the eyespot (Figure 7e), and the rotating filter system was constructed using the parameters given in Figure $7 \mathrm{~b}$. Our simulation of a spheroid swimming with rotation matches the in vivo situation in somatic cells very well (Figure 7d, red line). Spheroids were gently trapped between a coverglass and a glass slide, and fluid streamlines were visualized using polystyrene beads. After a dark-light switch, the light intensity was changed periodically (1.2-s intervals) with peaks in the intensity. The relative length of the tracks of polystyrene beads decreased sharply within $\sim 300 \mathrm{~ms}$ after the dark-light switch (Figures $7 \mathrm{f}-\mathrm{h}$ ), and this period of minimal bead movement persisted for $\sim 2.5 \mathrm{~s}$, even though two peaks in light intensity occurred during this time (Figure 7i). These peaks only caused a few changes in the length of the tracks in regions 1 and 2 (Figures $7 \mathrm{f}$ and $7 \mathrm{~g}$ ). After this delayed reaction, which is consistent with the speed reduction and sinking of spheroids after a dark-light switch described above, the relative length of the bead tracks in all analyzed regions at the anterior pole changed with the same frequency as the fluctuations in the light intensity (Figures $7 \mathrm{f}-\mathrm{i}$ ). The minimal length of the tracks lagged behind the peaks in light intensity by $~ 300$ ms. Interestingly, when the light was turned off after the periodical light pulses, the relative length of the bead tracks persisted at the maximal level (Figures $7 \mathrm{f}-\mathrm{i}$ ). Although the bead tracks were generally longer in region 2 (Figure $7 \mathrm{a}$ and $7 \mathrm{~g}$ ), which is closer to the spheroid than regions 1 and 3 , the results from all three regions were quite similar. 


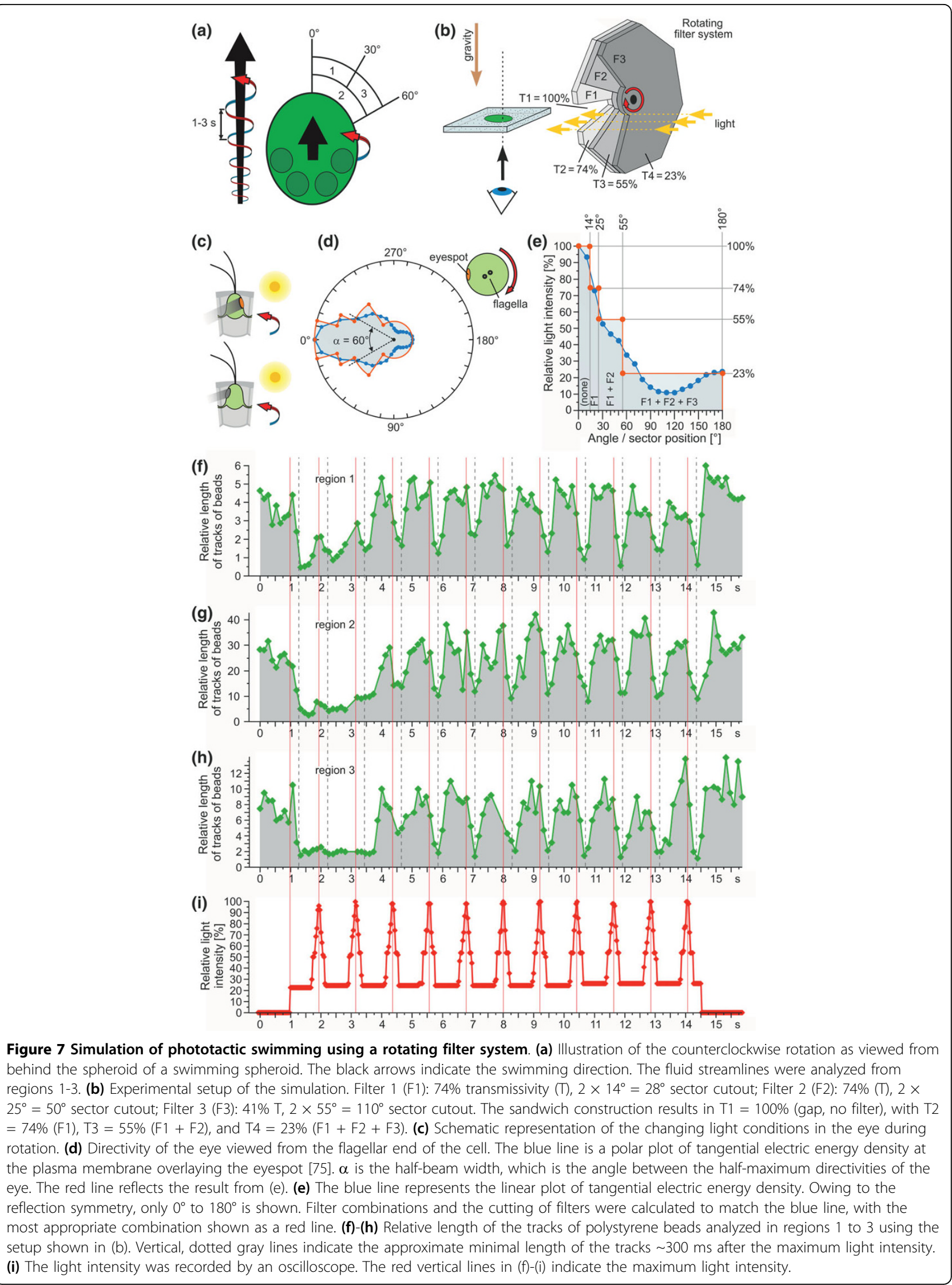




\section{Reaction time after light stimulation}

The reaction time of somatic cells at the anterior pole was calculated from high-speed microvideographs after a dark-light switch (Figures $2 \mathrm{~g}$ and $2 \mathrm{~h}$ ) and from darkfield microscopy with pulsed illumination (Figure 7). High-speed microvideography demonstrated that the flagella begin to reverse the beating direction after a dark-light switch after $\sim 100 \mathrm{~ms}$ (SD $25 \mathrm{~ms}, \mathrm{n}=5$ ). The transition from the normal beating mode to the reverse beating mode takes $\sim 30 \mathrm{~ms}$ (SD $10 \mathrm{~ms}, \mathrm{n}=5$ ). Thus, the period of time spanning the start of the light stimulation to the completion of the reversed beating direction is $\sim 100$ to $160 \mathrm{~ms}$. In the above experiments with periodically changing light intensities, the minimum of the fluid streamlines lagged behind the peaks in light intensity by $\sim 300 \mathrm{~ms}$ (Figures $7 \mathrm{f}-\mathrm{i}$ ). Owing to the reaction time of $\sim 100$ to $160 \mathrm{~ms}$, the reversed flagella need $\sim 140$ to $200 \mathrm{~ms}$ for their fluid streamlines to reach their minimum.

\section{Discussion}

In this study, we investigated key parameters of phototactic behavior in the spheroidal, multicellular volvocine green alga Volvox rousseletii, one of the fastest swimmers among all volvocine green algae. This is the first detailed description of phototactic behavior in a species within the Volvocales section Volvox.

\section{Light-induced reversal of the ciliary-type beating in Volvox rousseletii}

The reversal of the ciliary-type beating of the flagella shown in this study (Figure 2) has not yet been observed in other plants or green algae, but it has been reported for ciliates [76,77] and some metazoa $[78,79]$. In particular, the ciliate Paramecium caudatum has been widely known for its avoidance reaction, which is a sudden change in the swimming direction of the cell mediated by a transient reversal of its ciliary activity [80]. In Paramecium, the orientation of ciliary beating is controlled by the concentration of intracellular calcium [81-84]. Calcium is also well known for its role in the light-dependent responses of the unicellular Chlamydomonas, the best-studied volvocine alga with respect to flagellar activity and photoresponses [30,39,40,58,85-97]. The molecular mechanisms of light perception signal transduction and the control of flagellar beating identified in Chlamydomonas most likely are not specific to this species and instead are quite similar in all volvocine algae, given their close phylogenetic relationships. There are significant differences, however, in the mode of flagellar beating within the volvocine algae. Although the normal beating mode is a ciliary-type, asymmetrical motion, the light-induced flagellar beating pattern varies. Chlamydomonas responds with a flagellar-type, symmetrical beating, Volvox aureus [54] and V. carteri [55] respond with a cessation of flagellar beating, and $V$. rousseletii responds with a ciliary-type, asymmetrical beating in a reversed direction (Figure 2). These striking differences within the genus Volvox probably substantiate the polyphyletic origin of this genus. Phylogenetically, V. aureus and V. carteri belong to clade A within the Eudorina group [16,17], whereas $V$. rousseletii belongs to section Volvox [10,12,14-16] (see Additional File 1). Therefore, the light-induced ciliary-type, asymmetrical beating in a reversed direction might be characteristic of those species belonging to the section Volvox. The reversal of the beating direction in lightinduced cells should allow for faster changes in the swimming direction of the organism and better maneuverability compared with a simple cessation of flagellar beating in light-induced cells. Thus, $V$. rousseletii should outperform $V$. aureus and $V$. carteri in phototaxis merely because of its enhanced steering ability, not to mention its faster swimming speed. This assumption still requires experimental verification.

\section{Gravity facilitates positive phototaxis}

In addition to the flagellar forces, gravitational forces participate in phototaxis. Whenever a $V$. rousseletii spheroid slows down or stops swimming, gravity causes the spheroid to sink toward the bottom with its posterior pole first (see sections "Polarity of the $V$. rousseletii spheroid" and "Swimming speed after light stimulation as a function of the swimming direction" and Figure 5c). Even during normal swimming, gravity pulls on the posterior hemisphere more as a result of an anisotropic mass distribution caused by the denser daughter spheroids and probably by the closer spacing of somatic cells within the posterior hemisphere. This gravity effect has been quantitatively measured through biophysics in other species of the genus Volvox, which was referred to as "bottom-heaviness" [71,72]. Thus, through gravity, the posterior-anterior axis tends to become oriented in the opposite direction of the gravity vector such that the anterior pole tends to be on top. In the natural habitat, the sun, which serves as the light source, essentially shines from above, resulting in the direction of the light vector being roughly the same as that of the gravity vector. Therefore, gravity facilitates positive phototaxis because it brings the spheroids into the optimal orientation to swim toward the light source.

Even in the unicellular relative Chlamydomonas, cells with immobilized flagella sediment with their anterior ends pointing up $[98,99]$ because the posterior chloroplast-containing part of the cell body is denser than the anterior flagella-bearing part [100].

Thus, the utilization of gravity to ensure that the anterior end is always oriented toward the top while sinking 
seems to be a clear evolutionary advantage for all of the phototrophic and positively phototactic volvocine algae.

\section{Previous models on organismal steering in the genus Volvox}

In several earlier publications, observations in connection with phototaxis were combined to get an overall picture of how phototaxis works in Volvox. These descriptions and models of the swimming behavior in Volvox refer to the cells at the anterior hemisphere of the spheroid as being a crucial factor in phototactic steering and in its rotation during forward swimming to bring certain cells into or out of the light $[46,50,52-55,101]$. There had been different explanations as to how the individual cells on the anterior hemisphere change their flagellar activity in response to different light stimuli. Thus, the two above-mentioned competing models emerged, which were the variable beat frequency model $[48,52-55,57]$ and the variable beat direction model [46]. A more recent report analyzed both models and suggested the variable beat frequency model was the best description of phototactic turning in Volvox carteri [57]. This model is supported by the decrease of both the rate of progression and the rotation of $V$. carteri spheroids in the presence of illumination [57]. In addition, the fluid streamlines near the anterior pole of $V$. carteri [55] and $V$. aureus [52-54] spheroids stopped upon illumination. The researchers, however, did not monitor flagellar beating directly but drew conclusions from the behavior of those spheroids exposed to light stimuli. In 2006, Nozaki et al. [17] assigned the phylogenetic position of the species $V$. carteri and $V$. aureus to clade $\mathrm{A}$ in the Eudorina group (see Additional File 1).

The variable beat direction model [46] is supported by the following quite contrary, indirect observation: Mast [46] determined that the rate of progression of an unidentified species of the genus Volvox decreased after illumination while its rate of rotation increased. Mast's drawings and descriptions, however, indicated that the observed Volvox species belonged to the section Volvox [17]. Hoops et al. [57] tried but unfortunately could not replicate the step-up and step-down illumination experiments with Volvox rousseletii and Volvox capensis, both of which belong to the section Volvox. Therefore, Hoops et al. [57] concluded that a (re-)analysis of phototactic steering in the spheroids of the section Volvox is important.

The phylogenetic differences between the investigated species may indicate that there are differences in phototactic mechanisms that originally led to the two different models. Because the above models are based on experiments that do not include direct observations of flagella due to the small size and rapid movement of the flagella, the significance of these models may be limited.

\section{Mechanisms for phototactic steering in Volvox rousseletii: A model}

On the basis of the above and our own observations, we developed a mechanistic model that predicts the phototactic behavior in $V$. rousseletii. At the organismal level, we have shown that $V$. rousseletii spheroids respond to a sudden change in light intensity with a momentary photophobic response, reducing their speed rapidly for $\sim 2 \mathrm{~s}$ and causing a gravitational sinking of the spheroids (Figures 4b and 5). They then accelerate quickly and obtain their original swimming speed while turning phototactically toward the light source (Figure 5). In this response, not all flagellated somatic cells of the spheroid participate in the same way. There is a photoresponsive gradient from anterior to posterior, with the highest light sensitivity at the anterior pole of the spheroid and no response to light at the posterior pole (Figure 6). This gradient is reflected by an eyespot gradient in which the cells with the largest and most light-sensitive eyespots are located at the anterior pole and the cells with the smallest, even nonexistent, and blind eyespots are located at the posterior pole (Figure 1). After a change in light intensity, only cells in the anterior hemisphere with an adequately sized eyespot perceive the change in light intensity and switch transiently from the normal beating mode to a reverse beating mode (Figure 2 ), which is reflected by a momentary photophobic response at the cell level. The switch in the beating direction generates a force in the opposite direction on the anterior hemisphere, while cells on the posterior hemisphere still beat in the normal beating mode, causing a deceleration of the spheroid. The subsequent acceleration and phototactic turn toward the light source is complicated by the rotation of the spheroids in a counterclockwise direction as they swim along their posterior-anterior axis (Figure 7a). In addition, the eyes of the somatic cells show a strong directivity (Figure $7 d)$. Because the flagellar reaction cannot be observed in free swimming and rotating spheroids, the situation was simulated by holding the spheroid in place while the illumination was changed periodically (Figures $7 \mathrm{f}-\mathrm{i}$ ). Thus, the individual somatic cells of the anterior hemisphere are in the same situation as the corresponding somatic cells of a free swimming, rotating spheroid, which is illuminated on the side. The somatic cells of the anterior hemisphere respond quickly and periodically by reversing the direction of the flagellar beat (Figs. 2 and 7). In a free swimming, rotating spheroid, the local reversal of the beating direction causes an unbalanced force between the illuminated and the shaded side of the spheroid. As a result, the spheroid 
turns toward the light source. This mechanism does not require any communication between the cells because the flagellar responses of the somatic cells are coordinated only via the environmental factors of the position of the light source combined with the effects of gravity.

One final point that should be mentioned is that while each individual somatic cell has a forward and a reverse gear (Figure 2), the whole organism only has a forward gear, such that it can only swim in a posterior to anterior direction (Figure 6) but can turn easily.

The main components of our model of phototactic movements in $V$. rousseletii are illustrated in Figure 8, and the following conclusions summarize the relevant aspects.

\section{Conclusions}

On the basis of all results presented herein, we developed a mechanistic model that predicts phototactic behavior in Volvox rousseletii and shows how this multicellular organism achieves coordination among its individual biflagellated cells without direct communication between these cells.

The model encompasses the 10 following points:

1. The $V$. rousseletii spheroids (under the conditions of this study) show only positive phototaxis but not negative phototaxis, even at high light intensities (Figure 3).

2. Spheroids swim along the posterior-anterior axis and simultaneously rotate about this axis in a counterclockwise direction, with one turn every 1 to 3 seconds (Figure 7a).

3. The spheroid has a distinctive morphological polarity along the anterior-posterior axis, with only the somatic cells at the anterior pole having large eyespots $(\sim 3.6-\mu \mathrm{m}$ diameter $)$ and a continuous decrease in the eyespot size toward the posterior pole (Figure 1).

4. The spheroids show a strong photoresponse gradient from the anterior to the posterior pole, and only cells in the anterior hemisphere show an effective light response (Figure 6).

5. In response to light stimuli, the somatic cells of the anterior hemisphere can reverse and recover their flagellar beating mode quickly, within 100-160 ms (Figure 2).

6. The flagella show a ciliary-type, asymmetrical beating in both directions, but the flagella waveform and beat frequencies are different (Figure 2).

7. Gravity facilitates positive phototaxis due to the anisotropic mass distribution with the posterior of the spheroid being heavier than the anterior that results in the anterior, which contains the larger eyespots, always facing the top, where the light source normally is located (Figures $8 \mathrm{a}-\mathrm{c}$ ).

8. A sudden increase in light intensity causes the somatic cells of the anterior hemisphere to show a photophobic reaction for $\sim 2 \mathrm{~s}$, with an accompanying transient reversal of the ciliary activity (Figures $2 \mathrm{c}$ and $2 \mathrm{~h}$; Figure $6 \mathrm{~b}$ ), causing a deceleration and sinking of the spheroid (Figures 4, 5, and 8b).

9. During phototactic swimming, only somatic cells of the anterior hemisphere with eyespots pointing toward the light source reverse the beating direction in the presence of illumination, resulting in a turn toward the light source (Figures 2, 6, 7, and 8c).

10. The coordination of the spheroid's individual biflagellated cells during phototactic swimming occurs via environmental factors (e.g., the position of the light source and the effects of gravity) without any direct communication between the cells.

On the basis of our model of phototactic movements of the multicellular alga $V$. rousseletii, and taking into account previous considerations by Hoops [29] and Kirk [6], we conclude that the following evolutionary innovations were required to allow for phototactic movements after the transition from a unicellular Chlamydomonaslike ancestor to the multicellular $V$. rousseletii:

1. Both flagella of all cells must beat in essentially the same direction rather than the previously used breaststroke.

2. An extracellular matrix must hold all cells of the multicellular organism in the appropriate places on the surface of a spheroid.

3. The multicellular organism must not consist of identical cells and requires a distinctive morphological polarity, the flagella of all cells must beat toward the posterior, and the anterior cells must show a stronger light sensitivity, mediated through larger eyespots, than posterior cells.

4. The flagellar beating must cause rotation of the spheroid instead of rotation of the cell.

5. Light stimuli must cause a transient ciliary-type, asymmetrical beating in the reverse direction instead of a flagellar-type, symmetrical beating.

More work is clearly necessary, however, to confirm these evolutionary considerations.

\section{Methods}

\section{Strain and culture conditions}

The wild type Volvox rousseletii strain MI01 was grown in VT $[102,103]$ or VTAC $[104,105]$ medium at $20^{\circ} \mathrm{C}$ in a $10 \mathrm{~h}$ dark/14 h cycle under cool fluorescent white 


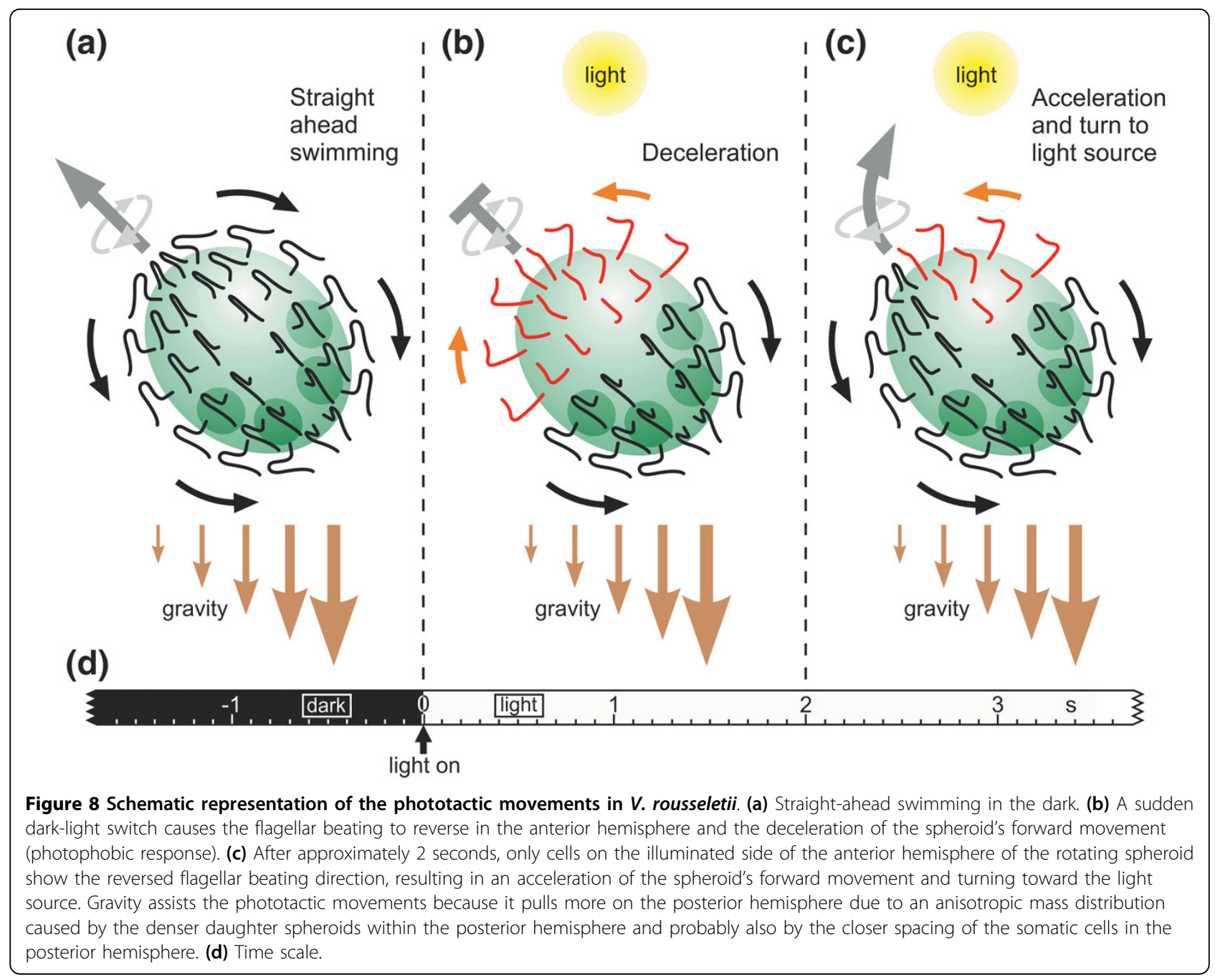

light at an average of $30-40 \mu \mathrm{mol}$ photons $\mathrm{m}^{-2} \mathrm{~s}^{-1}$ of photosynthetically active radiation.

\section{Analysis of photomovements and tracking of Volvox spheroids}

An observation chamber with internal dimensions of $10 \times 10 \times 1 \mathrm{~mm}$ was constructed as follows: (1) an acrylic cuvette (Sarstedt, Nümbrecht, Germany) was cut into slices with a 1-mm thickness, (2) a slice of the cuvette was glued onto a standard glass slide, and (3) the chamber was filled with a suspension of algae and covered with a coverglass, which was held in place by the adhesion force of water. The observation chamber contained 20-60 spheroids when it was used in the vertical orientation (Figure 3a) and 50-80 spheroids when in the horizontal orientation (Figure $3 \mathrm{~b}$ ). For observation in the vertical orientation (Figure 3a), the chamber was mounted upright on a stand and illuminated with a slide projector (Master Auto Lux-H; Rikagaku Seiki, Tokyo, Japan), and the movement of spheroids was recorded with a charge-coupled device (CCD) camera (XC-77; Sony, Tokyo, Japan) equipped with a C-mount macroscopic lens (Cine-Nikkor 1: 1.8, $\mathrm{f}=25 \mathrm{~mm}$; Nikon, Tokyo, Japan). For observation in the horizontal orientation (Figure $3 \mathrm{~b}$ ), the observation chamber was monitored using the CCD camera (XC77; Sony) on a standard inverted microscope (TMS-F; Nikon). A phototactically neutral field illumination was achieved with a long-pass filter $\left(\lambda_{\mathrm{T}}>750 \mathrm{~nm}\right.$, LX902 AcryFilter IR; Mitsubishi Rayon, Tokyo, Japan), which lets infrared light through but filters out all visible light. For the analysis of photomovement, the observation chamber was illuminated from the direction that was being tested (Figures $3 \mathrm{a}$ and $3 \mathrm{~b}$ ) using a continuous-output 500-W Xenon short-arc lamp (Ushio Electric, Tokyo, Japan) equipped with an interference filter for microscopic phase contrast illumination $\left(\mathrm{GIF}, \lambda_{\mathrm{T}(\max )}: 540 \mathrm{~nm}\right.$, spectral width [full width at half maximum]: $41 \mathrm{~nm}$; Nikon), which results in green light. 
The influence of light intensity on photomovements was analyzed using the 500-W Xenon short-arc lamp and a set of neutral density (ND) filters (ND1, ND3, ND10, ND20, and ND40; Hoya Corporation, Tokyo, Japan). Eight different light intensities $(0.00017 \mu \mathrm{mol} \mathrm{m}$ ${ }^{2} \mathrm{~s}^{-1}, 0.0017 \mu \mathrm{mol} \mathrm{m}{ }^{-2} \mathrm{~s}^{-1}, 0.017 \mu \mathrm{mol} \mathrm{m}{ }^{-2} \mathrm{~s}^{-1}, 0.16 \mu \mathrm{mol}$ $\mathrm{m}^{-2} \mathrm{~s}^{-1}, 2.18 \mu \mathrm{mol} \mathrm{m} \mathrm{s}^{-2} \mathrm{~s}^{-1}, 19.59 \mu \mathrm{mol} \mathrm{m}{ }^{-2} \mathrm{~s}^{-1}, 191 \mu \mathrm{mol}$ $\mathrm{m}^{-2} \mathrm{~s}^{-1}$ and $457 \mu \mathrm{mol} \mathrm{m} \mathrm{m}^{-2} \mathrm{~s}^{-1}$ ) were used in the experiments with the vertically oriented observation chamber, and 17 different light intensities $\left(0.00052 \mu \mathrm{mol} \mathrm{m}^{-2} \mathrm{~s}^{-1}\right.$, $0.00156 \mu \mathrm{mol} \mathrm{m} \mathrm{m}^{-1}, 0.0052 \mu \mathrm{mol} \mathrm{m}{ }^{-2} \mathrm{~s}^{-1}, 0.0156 \mu \mathrm{mol}$ $\mathrm{m}^{-2} \mathrm{~s}^{-1}, 0.052 \mu \mathrm{mol} \mathrm{m}{ }^{-2} \mathrm{~s}^{-1}, 0.156 \mu \mathrm{mol} \mathrm{m}{ }^{-2} \mathrm{~s}^{-1}, 0.52$ $\mu \mathrm{mol} \mathrm{m}{ }^{-2} \mathrm{~s}^{-1}, 1.04 \mu \mathrm{mol} \mathrm{m} \mathrm{m}^{-1}, 1.56 \mu \mathrm{mol} \mathrm{m}^{-2} \mathrm{~s}^{-1}, 2.08$ $\mu \mathrm{mol} \mathrm{m}{ }^{-2} \mathrm{~s}^{-1}, 5.2 \mu \mathrm{mol} \mathrm{m} \mathrm{s}^{-1}, 10.4 \mu \mathrm{mol} \mathrm{m} \mathrm{m}^{-2} \mathrm{~s}^{-1}, 15.6$ $\mu \mathrm{mol} \mathrm{m} \mathrm{s}^{-1}, 20.8 \mu \mathrm{mol} \mathrm{m} \mathrm{s}^{-2} \mathrm{~s}^{-1}, 52 \mu \mathrm{mol} \mathrm{m}{ }^{-2} \mathrm{~s}^{-1}, 208$ $\mu \mathrm{mol} \mathrm{m} \mathrm{m}^{-2} \mathrm{~s}^{-1}$ and $520 \mu \mathrm{mol} \mathrm{m} \mathrm{m}^{-2} \mathrm{~s}^{-1}$ ) were used in the experiments with the horizontally oriented observation chamber.

Phototactic movements were quantified by calculating the PI using the formula PI $=(B-A) \times 100 /(100-A)$, in which $A$ is the percentage of spheroids located in the investigated area (e.g., area 1) at the moment when the light was turned on $(0 \mathrm{~s})$ and $\mathrm{B}$ is the percentage of spheroids located in the same area at the respective time after the light was turned on. A high PI reflects a strong photoaccumulation within the given area.

Where applicable, individual images of recorded photomovements were analyzed with respect to the relative distribution of the spheroids in the chamber, the direction of the movements and the velocity of the movements using the NIH Image version 1.62 software [106].

\section{Recording of fluid streamlines}

The fluid streamlines generated by flagella were visualized and recorded according to the method of Hand and Haupt [53], with minor modifications. Briefly, $\sim 40$ $\mu \mathrm{l}$ of a suspension of individual Volvox spheroids in VT medium was pipetted onto a glass slide, and then 1-5 $\mu \mathrm{l}$ of an aqueous suspension of polystyrene beads (1- $\mu \mathrm{m}$ diameter; Polysciences, Warrington, PA, USA) were added. Two layers of standard vinyl tape were used as spacers between the slide and the coverglass to avoid crushing the spheroids, and the samples were analyzed on an inverted TMS-F microscope (Nikon). Infrared light was used for dark-field illumination, and green light was used for the stimulation of photomovements as described above. The tracks of the polystyrene beads were recorded with a CCD camera (C5985; Hamamatsu Photonics, Hamamatsu, Japan). Both the moving direction and speed of the fluid streamlines were calculated from the tracks of the polystyrene beads on the images.

\section{Construction and use of a rotating filter system for pulsed light stimulation}

A rotating filter system for pulsed light stimulation was constructed on the basis of the data of Foster and Smyth $[28,75]$ using three 2-mm thick acrylic filters, F1 (\#700; Sumitomo Chemical, Tokyo, Japan), F2 (\#700, Sumitomo Chemical), and F3 (\#702; Sumitomo Chemical), with transmissivities for green light of $74.4 \%$, $74.4 \%$, and $40.77 \%$, respectively. Sectors of $28^{\circ}$ (F1), $50^{\circ}$ (F2) and $110^{\circ}$ (F3) were cut out from the filters as calculated in Figure $7 \mathrm{e}$, and the sandwich construction was mounted reflection-symmetrically on a DC servomotor as shown in Figure $7 \mathrm{~b}$. The sandwich construction resulted in four different transmissivities: $\mathrm{T} 1=100 \%$ (gap, no filter); $\mathrm{T} 2=74 \%(\mathrm{~F} 1), \mathrm{T} 3=55 \%(\mathrm{~F} 1+\mathrm{F} 2)$, and $\mathrm{T} 4=23 \%(\mathrm{~F} 1+\mathrm{F} 2+\mathrm{F} 3)$. The filter system was rotated at $0.83 \mathrm{rps}(49.8 \mathrm{rpm})$. The rotating filter system was placed into the light path of the green light that was used to stimulate the photomovements. The oscillating light intensity was recorded by a silicon photodiode sensor (S1336-8BQ; Hamamatsu Photonics) connected to an oscilloscope (Tektronix, Beaverton, OR); the highest recorded light intensity $\left(28 \mu \mathrm{mol} \mathrm{m} \mathrm{m}^{-2} \mathrm{~s}^{-1}\right)$ was set to $100 \%$.

\section{Analysis of flagellar waveforms}

Flagellar waveforms were analyzed both with the CCD camera on the TMS-F microscope as described above using a long-pass filter with $\lambda_{\mathrm{T}}>610 \mathrm{~nm}$ for field illumination, and by high-speed microvideography as reported by Inouye and Hori [107]. In the latter case, flagellar activity (at $20^{\circ} \mathrm{C}$ ) was filmed at 200 frames per second using an MHS-200 high-speed video system (NAC Image Technology, Simi Valley, CA) attached to an Optiphoto light microscope (Nikon). The built-in strobe light of the high-speed video system was used to illuminate the field of view. Because the strobe light is indispensable for high-speed microvideography, the normal beating mode was recorded either right at the moment when the dark-adapted spheroids were illuminated with the strobe light, which corresponded to beating in the dark as the cells had no time to react, or after $10 \mathrm{~s}$ of illumination with the strobe light, which was several seconds after cells had adapted to the light.

\section{Analysis of eyespot sizes}

The spheroids were flattened gently by placing a suspension of algae between a slide (without vinyl tape spacers) and a coverglass. The flattened spheroids were observed with bright-field illumination using an upright compound microscope (BHS; Olympus, Tokyo, Japan). The digital images were analyzed using NIH Image software to calculate the eyespot size, defined as the largest 
diameter of the eyespots, and to determine the linear distance between a given eyespot and the anterior pole [106]. The anterior pole of the spheroid was identified as described earlier [5].

\section{Additional material}

\section{Additional file 1: Phylogenetic relationship between Volvox} rousseletii MI01 and other volvocine algae. This analysis is based on a combined data set of the psaA, psaB, and rbcL cDNA fragments from 47 volvocine species and/or strains. The unrooted tree was calculated by the neighbor-joining method using the PHYLIP software.

Additional file 2: Description of the phylogenetic analysis of the Volvox rousseletii strain MI01 that was utilized in this study. The Volvox rousseletii strain MI01 utilized in this study was subjected to a molecular phylogenetic analysis. To do so, certain DNA fragments that were used in the phylogenetic analyses of other volvocine algae were cloned and sequenced, which included the chloroplast genes encoding the photosystem I P700 chlorophyll a apoprotein A1 (psaA), the photosystem I P700 chlorophyll a apoprotein A2 (psaB), ribulose bisphosphate carboxylase (rbcL) and the internal transcribed spacer sequence 2 (ITS2).

Additional file 3: List of species, strains, abbreviations, accession numbers, and references for the $p s a \mathrm{~A}, p s a \mathrm{~B}, r b c \mathrm{~L}$ and ITS2 sequences from volvocine species.

Additional file 4: Sequence alignment of psaA cDNA fragments from several volvocine species.

Additional file 5: Sequence alignment of psaB cDNA fragments from several volvocine species.

Additional file 6: Sequence alignment of $r b c L$ CDNA fragments from several volvocine species

Additional file 7: Comparison of psaA sequences from several volvocine species

Additional file 8: Comparison of $p s a \mathrm{~B}$ sequences from several volvocine species.

Additional file 9: Comparison of $r b c \mathrm{~L}$ sequences from several volvocine species

Additional file 10: Comparison of ITS2 sequences from several volvocine species

\section{Abbreviations}

F: filter; n: sample size; Pl: photoaccumulation index; SD: standard deviation; T: transmissivity.

\section{Acknowledgements}

We thank Takeshi Nakayama and other members of the Inouye lab, as well as Terumitsu Hori (sadly now deceased) and members of his lab, for their useful discussions and technical suggestions. We also acknowledge a useful discussion with Hisayoshi Nozaki. Finally, we thank Kordula Puls and Mina Nomizu for their technical assistance. This work was supported in part by a grant from the Toyobo Biotechnology Foundation and by a JSPS

Postdoctoral Fellowship for Research Abroad (both to NU).

\section{Author details}

'Department of Cellular and Developmental Biology of Plants, University of Bielefeld, Universitätsstr. 25, 33615 Bielefeld, Germany. ${ }^{2}$ Institute of Biological Sciences, University of Tsukuba, Tsukuba, Ibaraki, 305-8577 Japan. ${ }^{3}$ The Promotion Center for Research and Education, Graduate University for Advanced Studies (Sokendai), Hayama, Kanagawa, 240-0193 Japan.

\section{Authors' contributions}

$\mathrm{NU}$ performed experiments and analyzed data. SM designed experiments, performed experiments, and contributed reagents and materials. II designed experiments and coordinated the first phase of the research project. $\mathrm{AH}$ (corresponding author) evaluated the data, coordinated the second phase of the research project, constructed the figures and wrote the paper. All of the authors read and approved the final manuscript.

Received: 10 May 2010 Accepted: 27 July 2010 Published: 27 July 2010

\section{References}

1. Szathmáry E, Smith JM: The major evolutionary transitions. Nature 1995, 374:227-232.

2. Bonner JT: The origins of multicellularity. Integr Biol 1998, 1:27-36.

3. Grosberg RK, Strathmann R: The evolution of multicellularity: a minor major transition? Annu Rev Ecol Evol Syst 2007, 38:621-654.

4. Prochnik SE, Umen J, Nedelcu A, Hallmann A, Miller SM, Nishii I, Ferris P, Kuo A, Mitros T, Fritz-Laylin LK, Hellsten U, Chapman J, Simakov O, Rensing SA, Terry A, Pangilinan J, Kapitonov V, Jurka J, Salamov A, Shapiro H, Schmutz J, Grimwood J, Lindquist E, Lucas S, Grigoriev IV, Schmitt R, Kirk D, Rokhsar DS: Genomic analysis of organismal complexity in the multicellular green alga Volvox carteri. Science 2010, 329:223-226.

5. Kirk DL: Volvox: molecular-genetic origins of multicellularity and cellular differentiation 1998.

6. Kirk DL: A twelve-step program for evolving multicellularity and a division of labor. BioEssays 2005, 27:299-310.

7. Herron MD, Hackett JD, Aylward FO, Michod RE: Triassic origin and early radiation of multicellular volvocine algae. Proc Natl Acad Sci USA 2009, 106:3254-3258.

8. Hoops HJ: Somatic cell flagellar apparatuses in two species of Volvox (Chlorophyceae). J Phycol 1984, 20:20-27.

9. Larson A, Kirk MM, Kirk DL: Molecular phylogeny of the volvocine flagellates. Mol Biol Evol 1992, 9:85-105.

10. Nozaki H, Itoh M, Sano R, Uchida H, Watanabe MM, Kuroiwa T: Phylogenetic relationships within the colonial Volvocales (Chlorophyta) inferred from rbcL gene sequence data. J Phycol 1995, 31:970-979.

11. Coleman AW: Phylogenetic analysis of "Volvocaceae" for comparative genetic studies. Proc Natl Acad Sci USA 1999, 96:13892-13897.

12. Nozaki H, Ohta N, Takano H, Watanabe MM: Reexamination of phylogenetic relationships within the colonial Volvocales (Chlorophyta): an analysis of $a t p B$ and $r b c L$ gene sequences. J Phycol 1999, 35:104-112.

13. Nozaki H, Takahara M, Nakazawa A, Kita Y, Yamada T, Takano H, Kawano S, Kato M: Evolution of $r b c \mathrm{~L}$ group IA introns and intron open reading frames within the colonial Volvocales (Chlorophyceae). Mol Phylogenet Evol 2002, 23:326-338.

14. Smith GM: A comparative study of the species of Volvox. Trans Am Microsc Soc 1944, 63:265-310.

15. Nozaki H, Ito M, Sano R, Uchida H, Watanabe MM, Takahashi H, Kuroiwa T: Phylogenetic analysis of Yamagishiella and Platydorina (Volvocaceae, Chlorophyta) based on rbcL gene sequences. J Phycol 1997, 33:272-278.

16. Nozaki H: Origin and evolution of the genera Pleodorina and Volvox (Volvocales). Biologia (Bratisl) 2003, 58:425-431.

17. Nozaki H, Ott FD, Coleman AW: Morphology, molecular phylogeny and taxonomy of two new species of Pleodorina (Volvoceae, Chlorophyceae). J Phycol 2006, 42:1072-1080.

18. Häder DP, Lebert M: Photoorientation in photosynthetic flagellates. Methods Mol Biol 2009, 571:51-65.

19. Nagel $G$, Ollig D, Fuhrmann M, Kateriya S, Musti AM, Bamberg E, Hegemann P: Channelrhodopsin-1: a light-gated proton channel in green algae. Science 2002, 296:2395-2398.

20. Sineshchekov OA, Jung KH, Spudich JL: Two rhodopsins mediate phototaxis to low- and high-intensity light in Chlamydomonas reinhardtii. Proc Natl Acad Sci USA 2002, 99:8689-8694.

21. Suzuki T, Yamasaki K, Fujita S, Oda K, Iseki M, Yoshida K, Watanabe M, Daiyasu H, Toh H, Asamizu E, Tabata S, Miura K, Fukuzawa H, Nakamura S, Takahashi T: Archaeal-type rhodopsins in Chlamydomonas: model structure and intracellular localization. Biochem Biophys Res Commun 2003, 301:711-717.

22. Nagel G, Szellas T, Huhn W, Kateriya S, Adeishvili N, Berthold P, Ollig D, Hegemann P, Bamberg E: Channelrhodopsin-2, a directly light-gated cation-selective membrane channel. Proc Natl Acad Sci USA 2003, 100:13940-13945. 
23. Zhang F, Prigge M, Beyriere F, Tsunoda SP, Mattis J, Yizhar O, Hegemann P, Deisseroth K: Red-shifted optogenetic excitation: a tool for fast neural control derived from Volvox carteri. Nat Neurosci 2008, 11:631-633.

24. Kianianmomeni A, Stehfest K, Nematollahi G, Hegemann P, Hallmann A: Channelrhodopsins of Volvox carteri are photochromic proteins that are specifically expressed in somatic cells under control of light, temperature, and the sex inducer. Plant Physiol 2009, 151:347-366.

25. Dieckmann CL: Eyespot placement and assembly in the green alga Chlamydomonas. BioEssays 2003, 25:410-416.

26. Schmidt M, Gessner G, Luff M, Heiland I, Wagner V, Kaminski M, Geimer S, Eitzinger N, Reissenweber T, Voytsekh O, Fiedler M, Mittag M, Kreimer G: Proteomic analysis of the eyespot of Chlamydomonas reinhardtii provides novel insights into its components and tactic movements. Plant Cell 2006, 18:1908-1930.

27. Hegemann P: Algal sensory photoreceptors. Annu Rev Plant Biol 2008, 59:167-189.

28. Kreimer $\mathrm{G}$ : The green algal eyespot apparatus: a primordial visual system and more? Curr Genet 2009, 55:19-43.

29. Hoops HJ: Motility in the colonial and multicellular Volvocales: structure, function, and evolution. Protoplasma 1997, 199:99-112.

30. Ringo DL: Flagellar motion and fine structure of the flagellar apparatus in Chlamydomonas. J Cell Biol 1967, 33:543-571.

31. Feinleib MEH, Curry GM: The relationship between stimulus intensity and oriented phototactic response (topotaxis) in Chlamydomonas. Physiol Plant 1971, 25:346-352.

32. Rüffer $U$, Nultsch W: High-speed cinematographic analysis of the movement of Chlamydomonas. Cell Motil 1985, 5:251-263.

33. Rüffer $U$, Nultsch W: Comparison of the beating of cis- and trans-flagella of Chlamydomonas cells held on micropipettes. Cell Motil Cytoskeleton 1987, 7:87-93

34. Rüffer U, Nultsch W: Flagellar photoresponses of Chlamydomonas cells held on micropipettes: I. Change in flagellar beat frequency. Cell Motil Cytoskeleton 1990, 15:162-167.

35. Rüffer U, Nultsch W: Flagellar photoresponses of Chlamydomonas cells held on micropipettes: II. Change in flagellar beat pattern. Cell Motil Cytoskeleton 1991, 18:269-278.

36. Polin M, Tuval I, Drescher K, Gollub JP, Goldstein RE: Chlamydomonas swims with two "gears" in a eukaryotic version of run-and-tumble locomotion. Science 2009, 325:487-490.

37. Takahashi T, Yoshihara K, Watanabe M, Kubota M, Johnson R, Derguini F, Nakanishi K: Photoisomerization of retinal at 13-ene is important for phototaxis of Chlamydomonas reinhardtii: simultaneous measurements of phototactic and photophobic responses. Biochem Biophys Res Commun 1991, 178:1273-1279.

38. Rüffer U, Nultsch W: Flagellar coordination in Chlamydomonas cells held on micropipettes. Cell Motil Cytoskeleton 1998, 41:297-307.

39. Hyams JS, Borisy GG: Isolated flagellar apparatus of Chlamydomonas: characterization of forward swimming and alteration of waveform and reversal of motion by calcium ions in vitro. J Cell Sci 1978, 33:235-253.

40. Bessen M, Fay RB, Witman GB: Calcium control of waveform in isolated flagellar axonemes of Chlamydomonas. J Cell Biol 1980, 86:446-455

41. Witman GB: Chlamydomonas phototaxis. Trends Cell Biol 1993, 3:403-408.

42. Gerisch G: Die Zelldifferenzierung bei Pleodorina californica Shaw und die Organisation der Phytomonadinenkolonien. Arch Protistenkd 1959, 104:292-358.

43. Hoops HJ, Floyd GL: Ultrastructure and development of the flagellar apparatus and flagellar motion in the colonial green alga Astrephomene gubernaculifera. J Cell Sci 1983, 63:21-41.

44. Hoops HJ: Flagellar, cellular and organismal polarity in Volvox carteri. J Cell Sci 1993, 104:105-117.

45. Greuel BT, Floyd GL: Development of the flagellar apparatus and flagellar orientation in the colonial green alga Gonium pectorale (Volvocales). J Phycol 1985, 21:358-371.

46. Mast SO: Reactions to light in Volvox, with special reference to the process of orientation. J Comp Physiol A Neuroethol Sens Neural Behav Physiol 1926, 4:637-658.

47. Linnaeus C: Systema naturae. Regnum animale. Stockholm , 101758.

48. Holmes SJ: Phototaxis in Volvox. Biol Bull 1903, 4:319-326.

49. Mast SO: Light reactions in lower organisms. II. Volvox globator. J Comp Neurol Psychol 1907, 17:99-180.
50. Mast SO: Light and the behavior of organisms New York: J. Wiley \& Sons, 1 1911.

51. Mast SO: The process of orientation in the colonial organism, Gonium pectorale, and a study of the structure and function of the eye-spot. $J$ Exp Zool 1916, 20:1-17.

52. Huth K: Bewegung und Orientierung bei Volvox aureus Ehrb. I. Mechanismus der phototaktischen Reaktion. Z Pflanzenphysiol 1970, 62:436-450.

53. Hand WG, Haupt W: Flagellar activity of the colony members of Volvox aureus Ehrbg. during light stimulation. J Protozool 1971, 18:361-364.

54. Sakaguchi H, Tawada K: Temperature effect on the photo-accumulation and phobic response of Volvox aureus. J Protozool 1977, 24:284-288.

55. Sakaguchi H, Iwasa K: Two photophobic responses in Volvox carteri. Plant Cell Physiol 1979, 20:909-916.

56. Sakaguchi H: Effect of external ionic environment on phototaxis of Volvox carteri. Plant Cell Physiol 1979, 20:1643-1651.

57. Hoops HJ, Brighton MC, Stickles SM, Clement PR: A test of two possible mechanisms for phototactic steering in Volvox carteri (Chlorophyceae). J Phycol 1999, 35:539-547.

58. Braun FJ, Hegemann P: Two light-activated conductances in the eye of the green alga Volvox carteri. Biophys J 1999, 76:1668-1678.

59. Ebnet $\mathrm{E}$, Fischer M, Deininger W, Hegemann P: Volvoxrhodopsin, a lightregulated sensory photoreceptor of the spheroidal green alga Volvox carteri. Plant Cell 1999, 11:1473-1484.

60. Solari CA, Ganguly S, Kessler JO, Michod RE, Goldstein RE: Multicellularity and the functional interdependence of motility and molecular transport. Proc Natl Acad Sci USA 2006, 103:1353-1358.

61. Drescher K, Goldstein RE, Tuval I: Fidelity of adaptive phototaxis. Proc Natl Acad Sci USA 2010, 107:11171-11176.

62. Meyer A: Die Plasmaverbindung und die Membranen von Volvox globator, aureus und tertius, mit Rücksicht auf die thierischen Zellen. Bot Zeitg 1896, 54:187-217.

63. Hoops HJ, Nishii I, Kirk DL: Cytoplasmic bridges in Volvox and its relatives. Cell-Cell Channels Georgetown, Texas: Eurekah.comBaluska F, Volkmann D, Barlow PW 2005, 1-20.

64. Schletz K: Phototaxis bei Volvox. Pigmentsysteme der Lichtrichtungsperzeption. Z Pflanzenphysiol 1976, 77:189-211.

65. Fritsch FE: The structure and reproduction of the algae. Vol. 1: Introduction Chlorophyceae, Xanthophyceae, Chrysophyceae, Bacillariophyceae, Cryptophyceae, Dinophyceae, Chloromonadineae, Euglenineae, colourless flagellata Cambridge, UK: Cambridge University Press 1935.

66. Coleman AW, Suarez A, Goff LJ: Molecular delineation of species and syngens in volvocacean green algae (Chlorophyta). J Phycol 1994, 30:80-90.

67. Mai JC, Coleman AW: The internal transcribed spacer 2 exhibits a common secondary structure in green algae and flowering plants. $\mathrm{J} \mathrm{Mol}$ Evol 1997, 44:258-271.

68. Coleman AW, Mai JC: Ribosomal DNA ITS-1 and ITS-2 sequence comparisons as a tool for predicting genetic relatedness. J Mol Evol 1997, 45:168-177.

69. Coleman AW, Preparata RM, Mehrotra B, Mai JC: Derivation of the secondary structure of the ITS-1 transcript in Volvocales and its taxonomic correlations. Protist 1998, 149:135-146.

70. Nozaki H, Misawa K, Kajita T, Kato M, Nohara S, Watanabe MM: Origin and evolution of the colonial Volvocales (Chlorophyceae) as inferred from multiple, chloroplast gene sequences. Mol Phylogenet Evol 2000, 17:256-268.

71. Pedley TJ, Kessler JO: Hydrodynamic phenomena in suspensions of swimming microorganisms. Annu Rev Fluid Mech 1992, 24:313-358.

72. Drescher K, Leptos KC, Tuval I, Ishikawa T, Pedley TJ, Goldstein RE: Dancing Volvox: Hydrodynamic bound states of swimming algae. Phys Rev Lett 2009, 102:168101.

73. Solari CA, Michod RE, Goldstein RE: Volvox barberi, the fastest swimmer of the Volvocales (Chlorophyceae). J Phycol 2008, 44:1395-1398.

74. Solari CA, Kessler JO, Michod RE: A hydrodynamics approach to the evolution of multicellularity: flagellar motility and germ-soma differentiation in volvocalean green algae. Am Nat 2006, 167:537-554.

75. Foster KW, Smyth RD: Light antennas in phototactic algae. Microbiol Rev 1980, 44:572-630. 
76. Nakamura S, Tamm SL: Calcium control of ciliary reversal in ionophoretreated and ATP-reactivated comb plates of ctenophores. J Cell Biol 1985 100:1447-1454.

77. Tamm SL, Terasaki M: Visualization of calcium transients controlling orientation of ciliary beat. J Cell Biol 1994, 125:1127-1135.

78. Mackie GO, Spencer AN, Strathmann R: Electrical activity associated with ciliary reversal in an echinoderm larva. Nature 1969, 223:1384-1385.

79. Galt CP, Mackie GO: Electrical correlates of ciliary reversal in Oikopleura. J Exp Biol 1971, 55:205-212

80. Naitoh $Y$, Eckert R: The control of ciliary activity in protozoa. Cilia and flagella London: Academic PressSleigh MA 1974, 305-352.

81. Naitoh $Y$, Eckert R: Ionic mechanisms controlling behavioral responses of Paramecium to mechanical stimulation. Science 1969, 164:963-965.

82. Naitoh $Y$, Eckert $R$, Friedman $K$ : A regenerative calcium response in Paramecium. J Exp Biol 1972, 56:667-681.

83. Nakaoka $Y$, Ooi H: Regulation of ciliary reversal in triton-extracted Paramecium by calcium and cyclic adenosine monophosphate. J Cell Sci 1985, 77:185-195.

84. Naitoh $\mathrm{Y}$, Kaneko $\mathrm{H}$ : Reactivated triton-extracted models of Paramecium: modification of ciliary movement by calcium ions. Science 1972 176:523-524

85. Stavis RL, Hirschberg R: Phototaxis in Chlamydomonas reinhardtii. J Cell Biol 1973, 59:367-377.

86. Stavis RL: The effect of azide on phototaxis in Chlamydomonas reinhardi. Proc Natl Acad Sci USA 1974, 71:1824-1827.

87. Schmidt JA, Eckert R: Calcium couples flagellar reversal to photostimulation in Chlamydomonas reinhardtii. Nature 1976, 262:713-715.

88. Litvin FF, Sineshchekov OA, Sineshchekov VA: Photoreceptor electric potential in the phototaxis of the alga Haematococcus pluvialis. Nature 1978, 271:476-478

89. Nultsch W: Effect of external factors on phototaxis of Chlamydomonas reinhardtii. III. Cations. Arch Microbiol 1979, 123:93-99.

90. Kamiya R, Witman GB: Submicromolar levels of calcium control the balance of beating between the two flagella in demembranated models of Chlamydomonas. J Cell Biol 1984, 98:97-107.

91. Omoto CK, Brokaw CJ: Bending patterns of Chlamydomonas flagella: II. Calcium effects on reactivated Chlamydomonas flagella. Cell Motil 1985, 5:53-60.

92. Harz H, Hegemann P: Rhodopsin-regulated calcium currents in Chlamydomonas. Nature 1991, 351:489-491.

93. Horst CJ, Witman GB: ptx1, a nonphototactic mutant of Chlamydomonas, lacks control of flagellar dominance. J Cell Biol 1993, 120:733-741.

94. Beck C, Uhl R: On the localization of voltage-sensitive calcium channels in the flagella of Chlamydomonas reinhardtii. J Cell Biol 1994, 125:1119-1125.

95. Pazour GJ, Sineshchekov OA, Witman GB: Mutational analysis of the phototransduction pathway of Chlamydomonas reinhardtii. J Cell Biol 1995, 131:427-440

96. Yoshimura K, Shingyoji C, Takahashi K: Conversion of beating mode in Chlamydomonas flagella induced by electric stimulation. Cell Motil Cytoskeleton 1997, 36:236-245.

97. Okita N, Isogai N, Hirono M, Kamiya R, Yoshimura K: Phototactic activity in Chlamydomonas 'non-phototactic' mutants deficient in $\mathrm{Ca}^{2+}$-dependent control of flagellar dominance or in inner-arm dynein. J Cell Sci 2005, 118:529-537

98. Kam V, Moseyko N, Nemson J, Feldman LJ: Gravitaxis in Chlamydomonas reinhardtii: characterization using video microscopy and computer analysis. Int J Plant Sci 1999, 160:1093-1098.

99. Yoshimura K, Matsuo Y, Kamiya R: Gravitaxis in Chlamydomonas reinhardtii studied with novel mutants. Plant Cell Physiol 2003, 44:1112-1118.

100. Kessler JO: The external dynamics of swimming microorganisms. Prog Phycol Res 1986, 4:257-307.

101. Huth K: Bewegung und Orientierung bei Volvox aureus Ehrb. II. Richtungsabweichung bei taktischen Reaktionen. Z Pflanzenphysiol 1970 63:344-351.

102. Provasoli L, Pintner IJ: Artificial media for fresh-water algae: problems and suggestions. The Ecology of Algae, a symposium held at the Pymatuning Laboratory of Field Biology on June 18 and 19, 1959 Pittsburgh, PA: The Pymatuning Symposia in Ecology, Special Publication No. 2., University of PittsburghTryon CA, Hartman RT , 1 1959, 84-96.
103. Watanabe MM, Nozaki H: NIES-collection: List of strains. Microalgae and protozoa Tsukuba, Japan: National Institute for Environmental Studies, 4 1994.

104. Nozaki H, Kuroiwa H, Mita T, Kuroiwa T: Pleodorina japonica sp. nov. (Volvocales, Chlorophyta) with bacteria-like endosymbionts. Phycologia 1989, 28:252-267.

105. Kasai F, Kawachi M, Erata M, Watanabe MM: NIES-collection: List of strains. Microalgae and protozoa Tsukuba, Japan: National Institute for Environmental Studies, 72004.

106. Rasband WS, Bright DS: NIH Image: a public domain image processing program for the Macintosh. Microbeam Anal Soc J 1995, 4:137-149.

107. Inouye I, Hori T: High-speed video analysis of the flagellar beat and swimming patterns of algae: possible evolutionary trends in green algae. Protoplasma 1991, 164:54-59.

doi:10.1186/1741-7007-8-103

Cite this article as: Ueki et al:: How 5000 independent rowers

coordinate their strokes in order to row into the sunlight: Phototaxis in the multicellular green alga Volvox. BMC Biology 2010 8:103.

\section{Submit your next manuscript to BioMed Central and take full advantage of:}

- Convenient online submission

- Thorough peer review

- No space constraints or color figure charges

- Immediate publication on acceptance

- Inclusion in PubMed, CAS, Scopus and Google Scholar

- Research which is freely available for redistribution

Submit your manuscript at www.biomedcentral.com/submit
C Biomed Central 